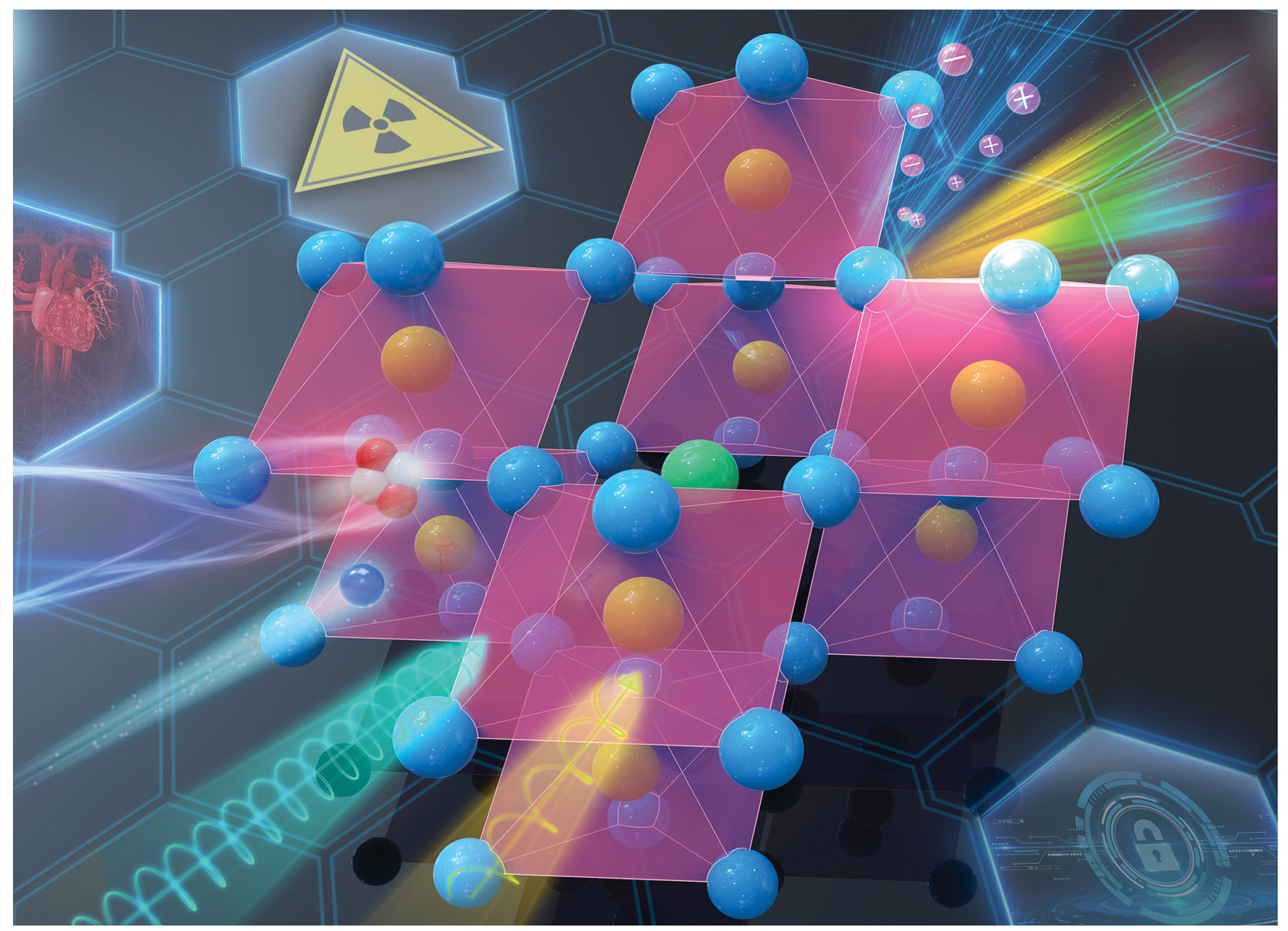

Showcasing research from Dr Feng Li's laboratory, School of Physics, The University of Sydney, Sydney, Australia.

Recent advances in radiation detection technologies enabled by metal-halide perovskites

The review aims at comprehensively summarizing the recent achievements, ongoing progress, and remaining challenges of metal halide perovskites for radiation detectors covering from high-energy particle radiations (alpha and beta particles) to photon radiations (gamma-ray and X-ray) and also propose a perspective on the opportunities offered by this emerging family on photoactive materials in the field of radiation technologies.

\section{As featured in:}

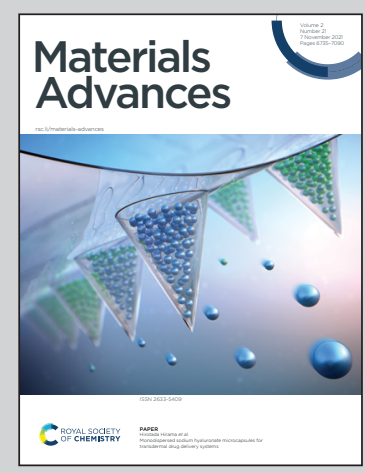

See Feng Li, Rongkun Zheng et al., Mater. Adv., 2021, 2, 6744. 
Check for updates

Cite this: Mater. Adv., 2021, 2,6744

Received 1st July 2021, Accepted 17th August 2021

DOI: $10.1039 / \mathrm{d} 1 \mathrm{ma} 00569 \mathrm{c}$

rsc.li/materials-advances

\title{
Recent advances in radiation detection technologies enabled by metal-halide perovskites
}

\begin{abstract}
Tiebin Yang, Feng Li (D)* and Rongkun Zheng*
Metal halide perovskites have emerged as promising candidates for next-generation optoelectronic applications due to their high absorption coefficient, tunable bandgap, long carrier mobility, and facile solution processability. Besides these excellent physical properties, their unique features, including large bulk resistance, strong stopping power, and high mobility-lifetime product, also favour halide perovskites for radiation detection. Their excellent radiation response and radioluminescence enable them as both direct detectors and indirect scintillators with excellent figures-of-merit, even exceeding the performance of existing commercial radiation detectors. In this review, the background knowledge on ionizing radiation categorized based on the nature of the particles or electromagnetic waves and the requirements for high-performance radiation detectors are provided first. Then, the relevant fundamental characteristics and superiorities of metal halide perovskites for radiation detection are discussed in detail. Thereafter, recent achievements in detecting alpha-, beta-, gamma- and X-rays using halide perovskites are summarised. Finally, the remaining challenges and future perspectives, with particular emphasis on overcoming current obstacles, such as device stability, ion migration, and health concerns, are highlighted.
\end{abstract}

\section{Introduction}

As a type of radiation that holds enough energy to ionize atoms or molecules, ionizing radiation has been widely applied in various areas in our life. ${ }^{1-3}$ In the form of particles or electromagnetic waves, ionizing radiation can be divided into directly

School of Physics, Australian Centre for Microscopy \& Microanalysis,

Nano Institute, the University of Sydney, NSW 2006, Australia.

E-mail: feng.li2@sydney.edu.au,rongkun.zheng@sydney.edu.au ionizing and indirectly ionizing, respectively. Any charged particle that has enough kinetic energy can ionize atoms through Coulomb force which induces direct ionizing. The directly ionizing radiation particles mainly include atomic nuclei, electrons, muons, charged pions, protons, and energetic charged nuclei stripped of their electrons, of which the most common types are alpha particles and beta particles that consist of helium nucleus and electrons or positrons, respectively. On the contrary, indirectly ionizing radiation is usually photon

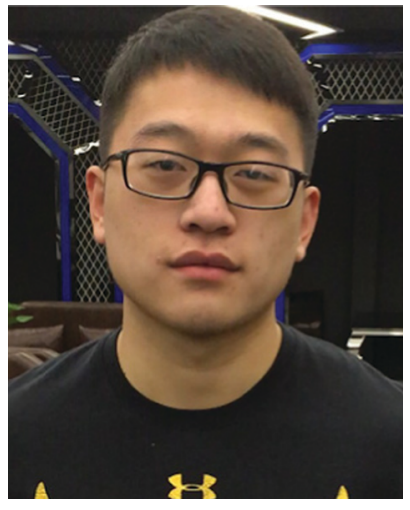

Tiebin Yang
Tiebin Yang is currently a PhD student at the School of Physics in the University of Sydney. He received his Bachelor's degree in Physics from Nanjing University in China in 2018. His research focuses on synthesis, characterization, and applications of all-inorganic halide perovskites.

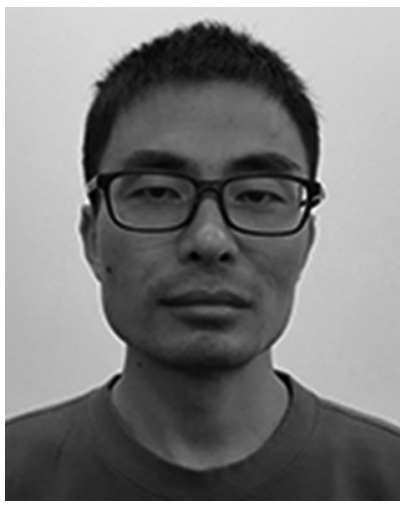

Feng Li
Feng $L i$ received his $P h D$ degree from the University of Chinese Academy of Sciences in 2014. He is currently a lecturer and a discovery early career researcher award (DECRA) fellow in the School of Physics at the University of Sydney. His main research is centered on the characterization and investigation of physical properties of functional materials, particularly hybrid perovskites and organic semiconductors, as well as diverse optoelectronic, energy and spintronic applications. 


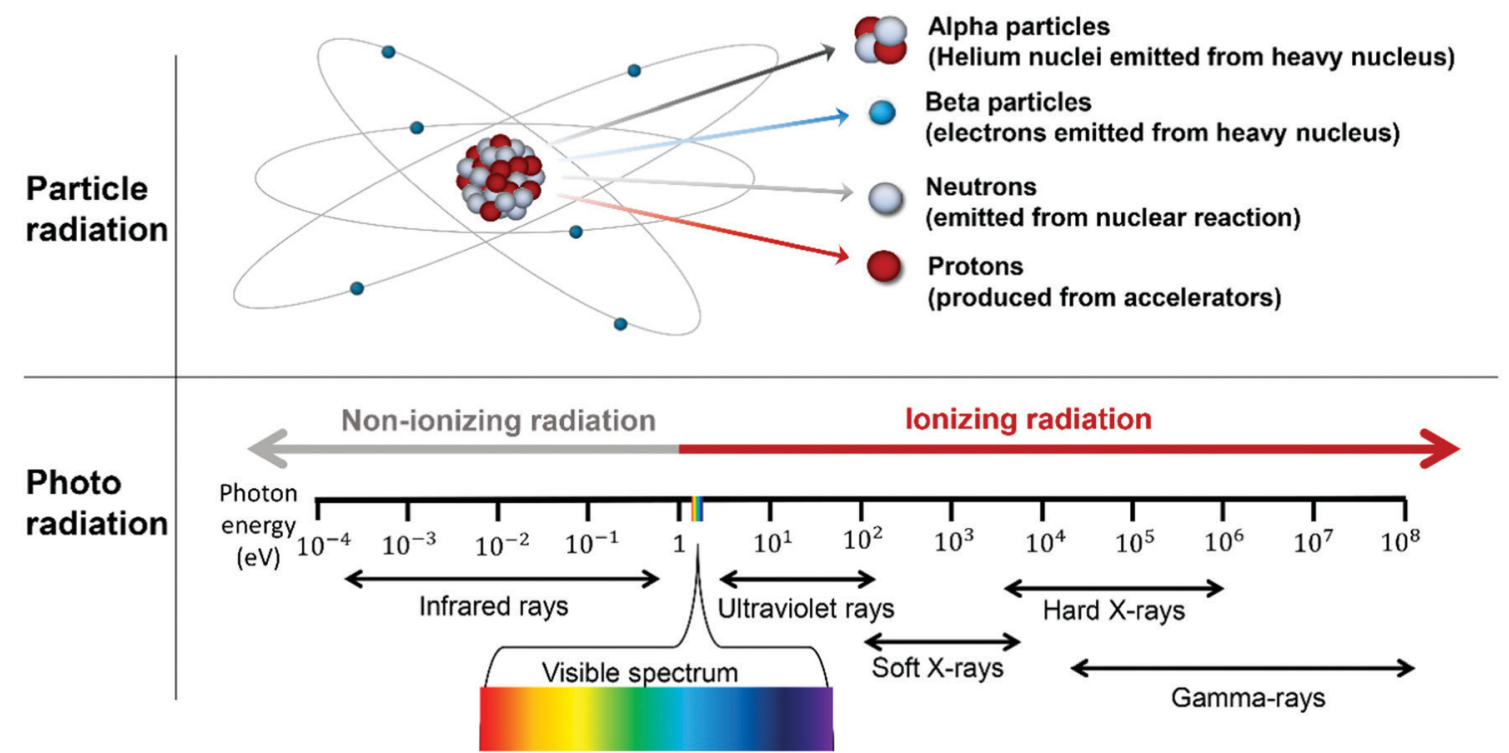

Fig. 1 Schematic representation of particle radiation with their formation (upper half), and the electromagnetic spectrum range from infrared to gamma-rays (lower half)

radiation or neutron radiation induced by electrically neutral particles, where the atoms will be further ionized by beta particles from the interaction of photons or neutrons with atoms. Photon radiation is typically known as high-energy electromagnetic waves, including X-rays (0.1-100 keV) and gamma-rays (0.1-100 MeV), which will cause ejection of electrons from atoms and then further ionize atoms by secondary beta particles, while neutrons often cause ionizing by interacting with protons in hydrogen via linear energy transfer. Fig. 1 clearly represents the formation of common radiation types, including particle radiation and photo radiation, and their related energies. While photo radiation can be divided into non-ionizing radiation and ionizing radiation, in our case, we mainly focus on ionizing radiation, including X-rays and gamma-rays. Thanks to their peculiarity of high penetration and ionization, radiation rays can be widely used in various fields, including nuclear physics,

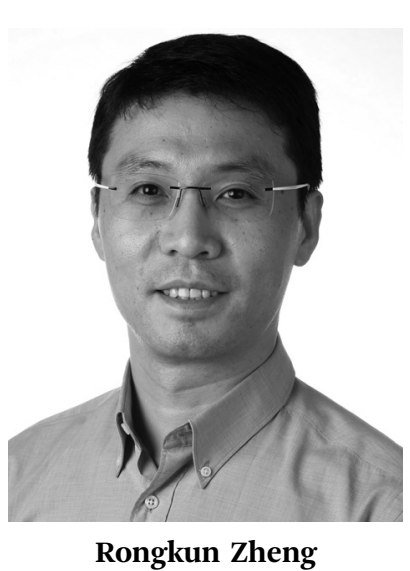

Rongkun Zheng obtained his BSc in Physics from Shandong University in China in 1999 and his PhD in Physics from the Hong Kong University of Science and Technology in 2004. He joined the University of Sydney in late 2004 and currently is a Professor in the School of Physics. His research interest spans from condensed matter and materials physics to microscopy and microanalysis, with focus on the growthstructure-property relationship in functional materials and devices. medical imaging, radiotherapy, food industry, security monitoring, crystallography, etc. ${ }^{4-8}$

In general, semiconductors with an appropriate bandgap can easily detect free charges induced by radiation under the applied voltages. Similar to the common photodetectors working in the UV-visible-infrared light ranges, semiconductor-based radiation detectors can also directly produce current signals from free-charged carriers excited by Coulomb force or through photoelectric effect. Current radiation detection devices are mainly based on silicon (Si), amorphous selenium (a-Se), germanium (Ge), cadmium zinc telluride (CZT), cadmium telluride (CdTe), and mercury iodide $\left(\mathrm{HgI}_{2}\right){ }^{9-12}$ However, some prerequisites need to be fulfilled for suitable semiconductors to serve as high-performance radiation detectors. First, the semiconductors' bandgaps are normally required to stay in the range from $1.5 \mathrm{eV}$ to $2.5 \mathrm{eV}$, which can ensure a low dark current at room temperature and provide enough energy barrier for electronhole pair production. Then, the high average atomic number $Z$ and high mass density lead to a large stopping power for successfully detecting high-energy radiation. For instance, even though the improvements of crystal purity and doping technique have been applied for the widely-used semiconductor $\mathrm{Si}$, the relatively small bandgap $(1.12 \mathrm{eV})$ causes a large dark current at room temperature and the small atomic number sets a great limitation for the fabrication of high-performance Si-based radiation detectors, while the CZT materials, with a relatively large bandgap ( $1.57 \mathrm{eV})$ and a high atomic number of 49.3 , have shown excellent performance in commercial radiation detectors, which can be operated at room temperature. ${ }^{13-15}$ Carrier mobility-lifetime product $\mu \tau$ is another important factor of suitable semiconductors when applied to radiation detection. A large $\mu \tau$ product indicates a long carrier diffusion length, by which the probability of a carrier being captured and recombined is reduced. Besides direct radiation detectors, radiation can also 
be detected using indirect detectors consisting of a scintillator and photodetector arrays. Scintillators can covert high-energy particles and photons into visible light via radioluminescence, which is further detected by other sensitive photodetectors such as charged-coupled devices (CCD), photodiode arrays, complementary metal-oxide semiconductors (CMOS), etc. Thus, the conversion efficiency highly determines the performance of the scintillator. To this end, high-quality scintillators should have the traits of a high light yield for generating enough detectable signals, long-term stability, and high energy resolution. Currently, the most commercially used scintillators are based on inorganic crystals, like thallium-doped sodium iodide ( $\mathrm{NaI}(\mathrm{Tl})$ ), thallium-doped caesium iodide (CsI(Tl)), and so on. ${ }^{16-18}$ However, these commercial semiconductors usually require complex growth methods and severe operating conditions, which create a sharp rise in the demand for new semiconducting materials with high performance, low cost and facile processing.

Over the past few years, as a family of promising photoactive materials, metal-halide perovskites with the general formula of $\mathrm{ABX}_{3}$, where the A site can be an inorganic cation like $\mathrm{Cs}^{+}$, a monovalent organic cation (typically a methylammonium $\left(\mathrm{MA}=\mathrm{CH}_{3} \mathrm{NH}_{3}\right)$ ion or a formamidinium $\left(\mathrm{FA}=\mathrm{CH}\left(\mathrm{NH}_{2}\right)_{2}\right)$ ion) or a mixture thereof, the $\mathrm{B}$ site is normally $\mathrm{Pb}$ or currently widely-researched Sn, and X is usually a halide component $\mathrm{Cl}-$, Br-, I-, or a mixture thereof, have attracted much attention in the photovoltaic field, ${ }^{19-21}$ due to their excellent features, including a long carrier lifetime, large absorption coefficient, high light yield, and cost-effective growth method. Looking beyond broadly-investigated halide perovskite solar cells, the superior photophysical and electronic properties of halide perovskites also enable them as promising candidates for next-generation optoelectronic or electronic device applications, such as light-emitting diodes, ${ }^{22-24}$ lasers,${ }^{25}$ transistors, ${ }^{26-29}$ and photodetectors. $^{30-33}$

Encouragingly, metal-halide perovskites have also been demonstrated to exhibit attractive features for radiation detection applications. Since Stoumpus et al. first pointed out that metal halide perovskites have the ability to detect radiations, ${ }^{34}$ many radiation detectors based on both hybrid perovskites and inorganic perovskites have also been successfully fabricated and further improved. ${ }^{35-38}$ In this regard, the rapid research progress and great strides that have been made by metal halide perovskites in ionizing radiation detection call for a swift and consistent survey into the state of the field. Notably, recently, there have been excellent review papers spanning from lead halide perovskites to lead-free perovskite with different crystal forms which cover fundamental physical properties and the related radiation detection devices. ${ }^{39-41}$ Each of these corresponding review publications has a different line of focus, depth, and narrative. However, the reviews centered on metal halide perovskite radiation detection applications that also include the detection of the directly ionizing radiation particles are limited. Therefore, this review paper aims to comprehensively summarize the recent achievements, ongoing progress, and challenges of halide perovskites for radiation detection devices with an emphasis on fundamental detection principles of various radiation photons and charged particles as well. We begin by offering the mechanism of different types of radiation detectors. The subsequent section comprehensively presents the unique physical characteristics of metal halide perovskite materials for radiation detection. Thereafter, the recent achievements and ongoing progress on metal-halide perovskite radiation detectors in mainly detecting alpha and beta particles, as well as gamma- and X-rays are summarised and discussed in detail. At last, we conclude this research article and present a perspective for the future development of halide perovskite materials for radiation detection applications and suggest some possible strategies to improve their long-term stability and device performance.

\section{Principles of radiation detectors}

\subsection{Direct radiation detectors}

Suitable semiconductors can directly detect radiation, of which the fabricated detectors can be operated in the current or voltage modes, depending on their direct interactions of incident particles or photons with the active semiconductors. ${ }^{42,43}$

2.1.1. Alpha and beta particle detections. For high-energy alpha particles, the direct radiation detectors usually work in the voltage mode, since the particle flux is relatively weak and alpha particles will come into the detector one by one. As the alpha particle interacts with the semiconductor via inelastic collisions, electron-hole pairs are generated whose number is proportional to the alpha particle energy. The electron-hole pairs generated by alpha particles are further collected and separated by the semiconductor under voltage bias in the form of current. However, the preliminary current signal intensity is relatively low, thus a charge-sensitive preamplifier is often integrated with the semiconductors. The amplifier then integrates the current signal and converts it into a voltage pulse which is proportional to alpha particle energy too. Finally, these voltage pulses are read out using a multiple channel digitizer with an alpha particle spectrum as the final output.

Compared to alpha particles, beta particles usually hold relatively lower energy but a much higher speed, which leads to a different interaction model with atoms. Beta particles can directly interact with other electrons in atoms by inelastic scattering, which excites other electrons to high-energy level or emitting photons. However, the beta particles can also interact with the nuclei through elastic scattering due to their much lower mass during which only the trajectories of the beta particles change. Once the electron-hole pairs are generated by beta particles, they can be detected by semiconductor-based detectors in the voltage mode too.

2.1.2. Gamma-ray detection. As for gamma-ray, an energyresolved spectrum can also be obtained by operating the direct radiation detectors in the voltage mode. ${ }^{44}$ But the electron-hole pairs can be generated in different ways depending on the different gamma-ray energies. A photoelectric effect often occurs when the gamma photon energy ranges from $10 \mathrm{keV}$ to $500 \mathrm{keV}$, where all the energies of gamma photons can be used for charged carrier generation. When the gamma-photon 
energies range from $50 \mathrm{keV}$ to $3 \mathrm{MeV}$, Compton scattering can happen from which some parts of energies from gamma photons will thus be transferred into electrons and/or holes. If gamma-rays hold energy over $\mathrm{MeV}$, electron and positron pairs will be generated in the active semiconductor which is called pair production. The $\mu \tau$ product is an important factor for gamma-ray detectors, which can be derived by fitting the modified Hecht formula: ${ }^{45}$

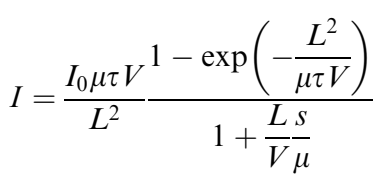

where $I_{0}$ is the saturated photocurrent, $L$ is the material thickness, $V$ is the applied voltage and $s$ is the surface recombination velocity. The gamma-ray detectors' performance can be typically characterized by the spectral peak resolution, which is defined by the ratio of full-width at half maximum (FWHM) and the incident energy of the radiation source.

2.1.3. Direct X-ray detection. Direct X-ray detectors can usually work in the current mode, where the photon flux is strong to generate enough current signals. Compared to highenergy particles or photons which are collected by the detector separately, multiple X-ray photons come and interact with the active semiconductors at the same time and are converted into electron-hole pairs by both photoelectric effect and Compton scattering. Many charged carriers are generated during these two processes, which will further be collected by the electrodes under the applied voltage bias to produce the current signals. The key parameter for direct X-ray detection is sensitivity, which is calculated by ${ }^{46}$

$$
S=\frac{\int\left(I_{\mathrm{X}-\mathrm{ray}}-I_{\mathrm{dark}}\right)}{\mathrm{d} t}
$$

where $I_{\mathrm{X} \text {-ray }}$ and $I_{\text {dark }}$ are current under X-ray radiation and in the dark condition, respectively, $D$ is the $\mathrm{X}$-ray dose rate and $V$ is detector volume. Detectors with high sensitivity can generate more current signals under the same irradiation with a higher signal-to-noise ratio, which leads to higher contrast when applied to imaging applications. The linear dynamic range (LDR) describes the range in which the sensitivity remains constant. A large LDR value means that the related detectors can work steadily among a large range of X-rays with different dose rates. Another important feature of the X-ray detector is the lowest detectable dose rate, which is the detection limit of an X-ray detector and is highly related to X-ray imaging in medical diagnostics. Spatial resolution characterizes the imaging quality obtained by an X-ray detector and is defined as the number of line pairs that can be distinguished per millimeter. Direct X-ray detectors often integrate with semiconductor photodiode arrays for imaging and therefore spatial resolution is affected by both photodetector and photodiode arrays.

\subsection{Indirect radiation detection}

The indirect radiation detectors are also capable of detecting high-energy particles or photons, and often consist of scintillator and photodiode arrays. ${ }^{47}$ As mentioned above, high-energy particles can ionize the active semiconducting materials by Coulomb force; while high-energy photons can interact with the materials via the photoelectrical effect, Compton scattering, and pair production. All these processes will generate many excitons in the scintillators which will be transferred to the defects states and further recombine together with UV or visible light-emitting effect. The emitted UV or visible light will be further captured by photo-sensitive photodetector arrays or cameras, and then the output is offered as a current signal or image information. The key figures of merit of scintillators are light yield (LY) and decay time. Light yield is the number of photons that can be converted by the scintillator per photon or particle energy (in unit), which can be calculated using ${ }^{1}$

$$
L Y=10^{6} S Q / \beta E_{\mathrm{g}}
$$

where $S$ is the efficiency of the transport of electron-hole pairs to the emission centre, $Q$ is the radioluminescence efficiency and $\beta$ is usually a constant of 2.5. A high LY value indicates the high number of photons emitted from the scintillator which leads to a high signal output. For a high-performance scintillator, a fast decay time is essential since the time interval between the emission of photons and absorption of radiation is short, which will reduce some side effects such as afterglow. Table 1 summarises some of the main figures of merit used to characterize and evaluate the radiation detectors in the different working models.

\section{Superb properties of halide perovskites for radiation detection}

As mentioned above, the superior photophysical and electronic properties of lead halide perovskites, including long carrier lifetimes, large absorption coefficients, high light yields, and cost-effective growth methods, ${ }^{48-52}$ enable them as promising candidates for next-generation optoelectronic devices, such as light-emitting diodes, ${ }^{53-56}$ lasers, ${ }^{57}$ solar cells ${ }^{58-61}$ and photodectors. ${ }^{62-67}$ Furthermore, several prerequisites need to be fulfilled for suitable semiconductors to serve as high-performance radiation detectors. Encouragingly, lead halide perovskites have exhibited excessive properties that are actually desirable for radiation detecting devices. Currently, there is an increasing number of published reports on radiation detectors based on both hybrid halide perovskites and inorganic perovskites that have also been fabricated for detecting alpha particles, beta particles, gamma-rays, and X-rays. ${ }^{40,41,68}$ In this section, the superb features of halide perovskite materials for radiation detection applications are introduced in detail as follows:

\subsection{Large stopping power}

Large stopping power is essential for the high-performance of radiation detectors, since more high-energy particles can be dragged down with more energy loss and thus more signal outputs. Given by the Bethe equation, ${ }^{69}$ the energy loss of charged particles passing through certain materials can be 
Table 1 Radiation detector definition and figures of merit

\begin{tabular}{|c|c|c|}
\hline Quantity & Unit & Definition \\
\hline Spectral peak resolution $^{a b}$ & $\%$ & $\begin{array}{l}\text { Defined by the ratio of full width at half maximum (FWHM) of alpha or gamma-ray peak and } \\
\text { energy of incident ray. }\end{array}$ \\
\hline Spatial resolution $^{a b}$ & $\mathrm{lp} \mathrm{mm^{-1 }}$ & $\begin{array}{l}\text { The number of line pairs that can be distinguished per millimetre. A line pair consists of a bright line } \\
\text { and a dark line (in X-ray imaging). }\end{array}$ \\
\hline Linear dynamic range $^{a}$ & & The range within which the photocurrent displays a linear relationship with the incident intensity. \\
\hline Light yield $^{b}$ & $\mathrm{ph} \mathrm{MeV}^{-1}$ & Number of photons generated by radiation with a specific energy. \\
\hline Decay time ${ }^{b}$ & s & $\begin{array}{l}\text { The time taken by the emitting light intensity excited by radiation to decay to } 1 / e \text { of its initial } \\
\text { intensity. }\end{array}$ \\
\hline
\end{tabular}

described as:

$$
-\frac{\mathrm{d} E}{\mathrm{~d} x}=\frac{4 \pi e^{4} z^{2}}{m_{0} v^{2}} N Z\left[\ln \frac{2 m_{0} v^{2}}{I}-\ln \left(1-\frac{v^{2}}{c^{2}}\right)-\frac{v^{2}}{c^{2}}\right]
$$

where $m_{0}$ and $v$ are the mass and velocity of the incoming charged particles, respectively; while $N$ and $Z$ are the number density and atomic number of the absorbing material, respectively. Heavy metal lead $(\mathrm{Pb})$ and halide components (usually $\mathrm{Br}$ or I) provide this class of perovskite materials with high average atomic numbers, which offer a large stopping power and high detection efficiency accordingly. For example, all-inorganic halide perovskite $\mathrm{CsPbBr}_{3}$ has an average atomic number of 65.9, which is even larger than that of 49.3 for the CZT material. Hybrid perovskite $\mathrm{MAPbI}_{3}$ has a density of $4 \mathrm{~g} \mathrm{~cm}^{-3}$, corresponding to a linear attenuation coefficient of $10 \mathrm{~cm}^{-1}$ at $100 \mathrm{keV}$, while all-inorganic perovskite $\mathrm{CsPbI}_{3}$ even holds a larger linear attenuation coefficient of $14 \mathrm{~cm}^{-1}$. Fig. 2a shows the linear attenuation coefficients of halide perovskite materials - $\mathrm{MAPbI}_{3}$ and $\mathrm{CsPbI}_{3}$ and that of the commonly-used Se, CdTe, and TlBr at different photon energies, from which we can find that the halide perovskite materials have linear attenuation properties comparable with these commonly-used materials and even higher than some semiconductors in the particular energy ranges.

\subsection{Suitable bandgap and large bulk resistance}

As mentioned above, appropriate bandgaps for the semiconducting materials are crucial for high-performance radiation detectors, especially for room-temperature operations. Encouragingly, lead halide perovskites hold tunable bandgaps ranging from 1.3 to $3.2 \mathrm{eV}$ that can be easily realized by adjusting their halide components, ${ }^{70-74}$ which make them perfectly suitable for radiation detectors (Fig. $2 \mathrm{~b}$ and c). Meanwhile, the bulk resistances of lead halide perovskites range from $10^{7}$ to $10^{9} \Omega \mathrm{cm}$, which can be further modified by tuning the halide component ratio of perovskites. The large bulk resistances ensure the low dark currents at room temperature and the low noises of the perovskite radiation detectors, especially when applied to a high voltage bias.

\subsection{High mobility-lifetime product}

Both the intrinsic and extrinsic defects of the semiconducting materials can capture the charge carriers that are generated by radiation, thus decreasing the signal currents of the related detection devices. Large carrier-lifetime product $\mu \tau$ values can reduce the recombination of electron-hole pairs at the defects and traps within them, and thus more charge carriers can be collected by the electrodes which further increases the efficiency of the detector. As for the halide perovskite films, most defect centres are located at the surface or grain boundaries. To this end, several surface cleaning and passivation approaches have been applied to reduce the radiation-generated carrier recombination and thus improve the film quality. ${ }^{75}$ Among various forms of halide perovskites, grain-boundary-free single crystals usually exhibit a low trap density in the range of $10^{7}-10^{9} \mathrm{~cm}^{-3}$ and thus offer high $\mu \tau$ products. $^{76-78}$ For example, hybrid perovskite $\mathrm{MAPbI}_{3}$ single crystal holds a high $\mu \tau$ product of $10^{-2} \mathrm{~cm}^{2} \mathrm{~V}^{-1}$, with an ultralong carrier diffusion length over $175 \mu \mathrm{m}$ under the illumination of sunlight. ${ }^{79}$ Moreover, the internal quantum efficiency of a $3 \mathrm{~mm}$ thick $\mathrm{MAPbI}_{3}$ single crystal can also be approaching $100 \%$ under weak light illumination, which indicates a diffusion length almost exceeding $3 \mathrm{~mm} .{ }^{79}$

\subsection{Facile and low-cost crystal growth method}

Other advantages of halide perovskite materials and the relevant device applications include their cost-effective crystal growth and device fabrication methods. Compared to the widely-used commercial semiconductors, like Si, Ge, Se, and $\mathrm{CZT}$, for which the growth processes typically require high temperature, high vacuum, or complex instruments, lead halide perovskites can usually be produced using the lowtemperature $\left(<150{ }^{\circ} \mathrm{C}\right)$ solution processes, such as inverse temperature crystallization (ITC), ${ }^{78,80}$ antisolvent vaporassisted crystallization (AVC), ${ }^{77,81}$ hot inject method, ${ }^{48}$ and supersaturated recrystallization. ${ }^{82,83}$ Moreover, the total cost for the growth of halide perovskite materials is quite low as compared to that of the commonly-used semiconductor as mentioned above. For example, growing a $1 \mathrm{~cm}^{3}$ perovskite single crystal is estimated to cost around $\$ \$ 0.3$ when scaling up the production, which is 3- or 4-times lower than that for CZT 


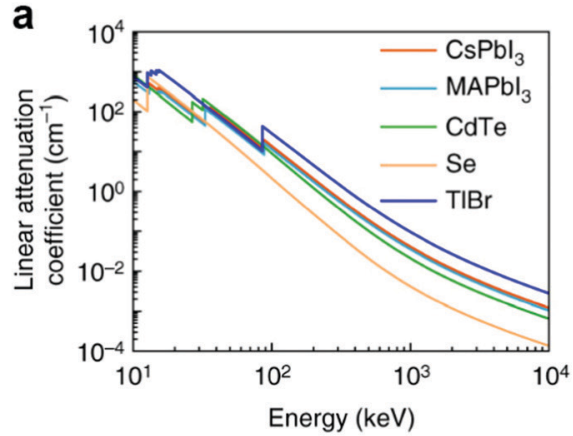

b
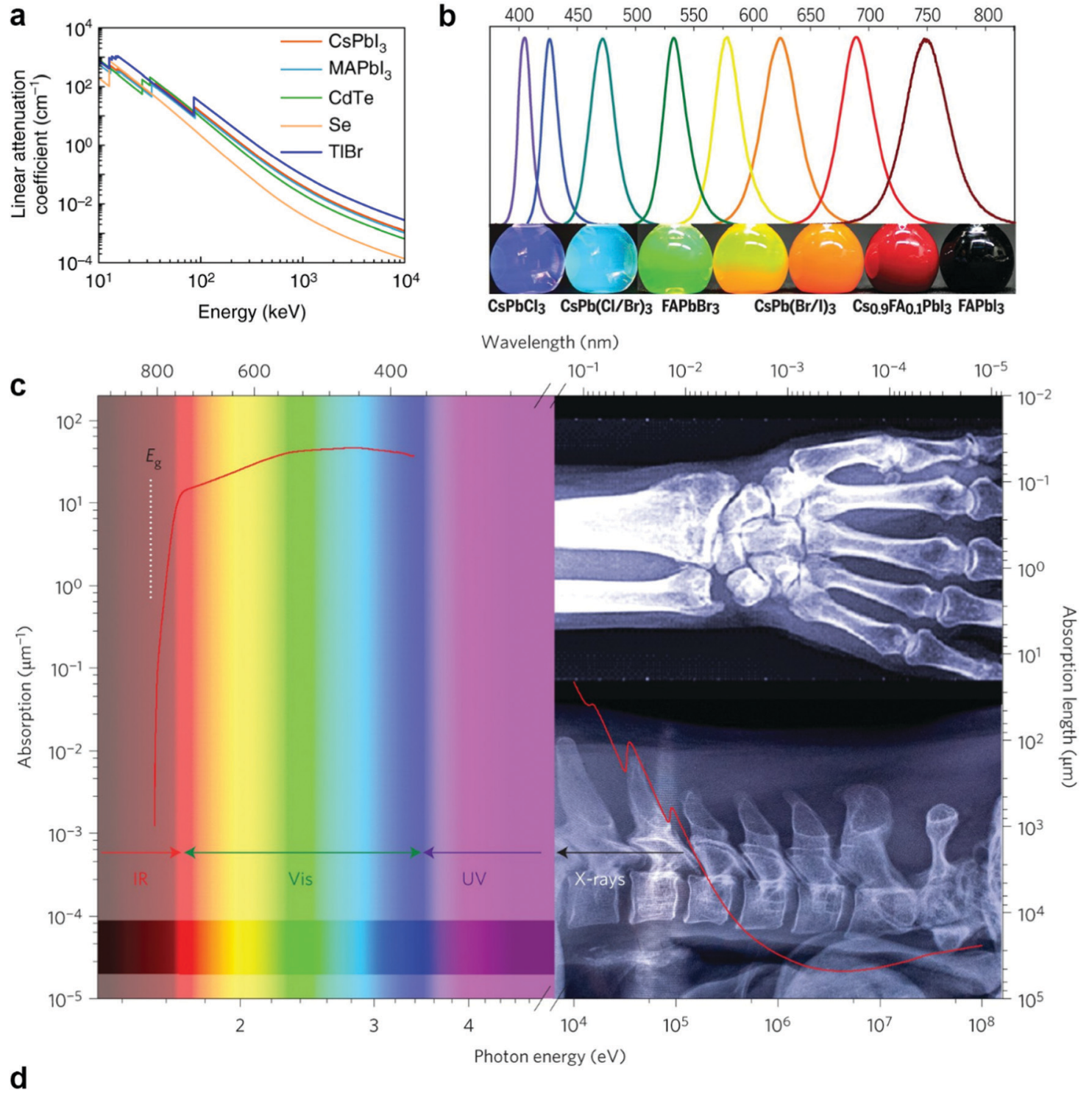

Wavelength (nm)

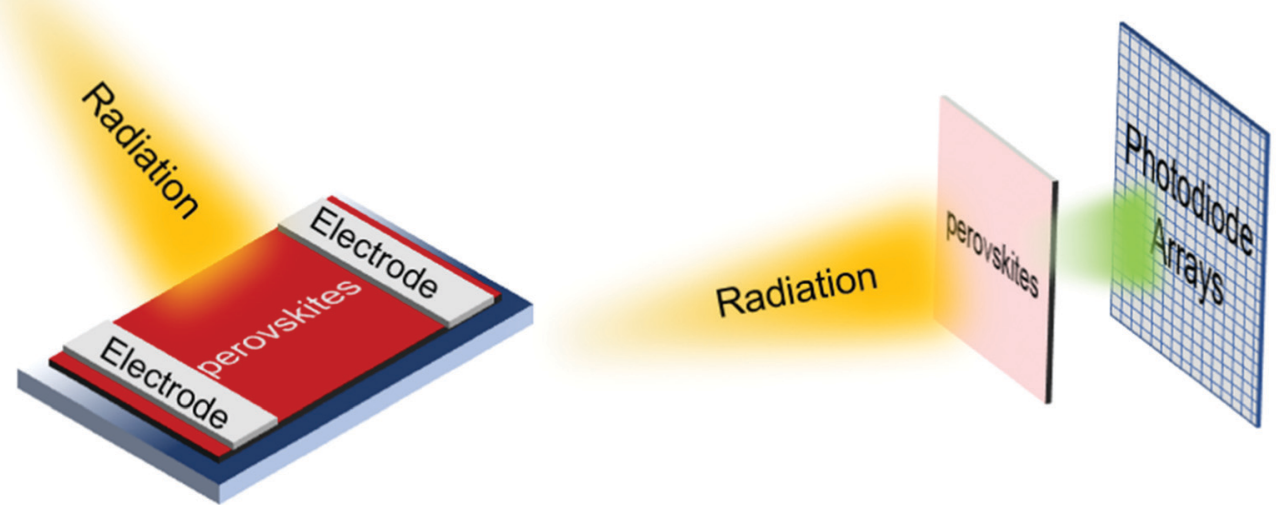

Fig. 2 (a) Attenuation coefficients of $\mathrm{CsPbl}_{3}, \mathrm{MAPb}_{3}, \mathrm{CdTe}, \mathrm{Se}$, and $\mathrm{TlBr}$ versus different photon energies. Reproduced with permission. ${ }^{39}$ Copyright 2019, Nature Publishing Group. (b) PL spectra of the tunable APbX 3 nanocrystals. Reproduced with permission. ${ }^{87}$ Copyright 2017, AAAS. (c) Absorption coefficient and length with different photo energies of $\mathrm{MAPb}_{3}$ crystals. Reproduced with permission. ${ }^{88}$ Copyright 2015, Nature Publishing Group. (d) Schematic of the structure of basic radiation detectors. Left: Direct detector. Right: Indirect scintillator. 
crystals. ${ }^{39}$ Besides, the related detection devices can be easily fabricated via a one-step spin-coating or spray method and directly using the freshly-grown perovskite crystals, which offers a large operation room for further integration.

\subsection{High light yield}

The fascinating properties of lead halide perovskites can also enable them as both direct photoconductors and indirect scintillators for radiation detection. When transforming into different crystal forms, like nanocrystals and quantum dots, lead halide perovskites exhibit strong radioluminescence under the excitation of high-energy radiations. For instance, as compared to $\mathrm{LaBr}_{3}-\mathrm{Ce}$, which exhibited optimal device performance with a light yield (LY) of $70000 \mathrm{ph} \mathrm{MeV}^{-1}$ and a decay time of $16 \mathrm{~ns}$, lead halide perovskites hold a much higher light yield (LY) over $10^{6} \mathrm{ph} \mathrm{MeV}^{-1}$ and a fast decay time less than $1 \mathrm{~ns}^{84}$ Such high light yield (LY) and fast decay time ideally meet the requirement for first-class scintillators, which enable them to measure the time of initial particle or radiation with high precision and timing resolution. Besides, halide perovskite nanocrystals also permit easy halide ion exchanges, from which a tunable luminescence spectrum across almost the whole visible region can be obtained. ${ }^{85,86}$

Armed with these unique advantages, direct radiation detectors based on halide perovskite single crystals and polycrystalline films have exhibited excellent device performance in a photoconductor mode. ${ }^{35,88-93}$ Fig. $2 d$ shows the common device structure of the halide perovskite direct radiation detector, which is composed of a perovskite layer and two electrodes in the left part. The right side in Fig. 2d displays the schematic diagram of the perovskite-based indirect detector which normally consists of a perovskite scintillator and another photodetector array. Excellent compatibility and easy fabrication methods also enlarge the room for further improving the device performance. Besides, due to their high light yield under radiation, lead halide perovskites can serve as scintillators integrated with other semiconductor-based photodiodes, by which high-performance radiation detectors can be achieved with multiple detection abilities. $^{94-96}$

\section{Charged particle detection by lead halide perovskites}

\subsection{Alpha-particle detectors}

In 1899 , the alpha particles were named by Rutherford as they have the lowest penetration of ordinary objects; and in 1907, these particles were then confirmed as helium nuclei. ${ }^{97}$ Emitted from the alpha decay of heavy atoms, alpha particle energy varies with the half-life of the emission process, where the higher energy is caused by the larger nucleus. Most alpha particles can hold energy ranging from 3 to $7 \mathrm{MeV}$, with a speed of $15000 \mathrm{~km} \mathrm{~s}^{-1}$ due to their higher particle mass as compared to other radiation rays. Thus, the penetration of the alpha particles is typically lower than that of beta particles or gamma rays, and can be blocked by a piece of paper or human skin.
However, it is still the most destructive ionizing radiation; if alpha-emitting atoms are inhaled, ingested, or injected into our body, they will cause serious chromosomal damage to DNA which is 20 times greater than that caused by the same dose of gamma-rays or beta particles. ${ }^{98}$ In this regard, developing highquality detectors for alpha particle radiation would be of significance, as they will be of use not only for environmental safety concerns but also for the nuclei information carried out by alpha particles. An alpha spectrum is commonly used to characterize alpha particles that are emitted from the nuclei. Thus peak resolution is an important parameter for alpha radiation detectors, which helps distinguish the alpha radiation peak in the alpha spectrum. As mentioned in the above section, another key parameter of alpha particle detectors is the $\mu \tau$ product, which is directly related to the charge collection efficiency (CCE) and can be derived from the Hecht equation for single carrier: ${ }^{99,100}$

$$
\eta=\frac{Q}{Q_{0}}=\frac{\mu \tau V}{d^{2}}\left(1-\mathrm{e}^{-\frac{d^{2}}{\mu \tau V}}\right)
$$

where $\eta$ is CCE, $Q$ and $Q_{0}$ are the maximum and theoretical channel numbers, $V$ is applied voltage, and $d$ is the thickness of the detector.

Recently, Xu et al. fabricated alpha particle detectors based on hybrid perovskite $\mathrm{MAPbBr}_{3}$ single crystals $(5 \mathrm{~mm} \times 5 \mathrm{~mm} \times$ $2 \mathrm{~mm}$ ) with a metal-semiconductor-metal structure. ${ }^{101}$ As shown in Fig. 3a, the organic BCP and $\mathrm{C}_{60}$ layers were coated for the functions of both passivation and electron extraction, and a preamplifier and a digitizer were used for alpha spectrum measurements. A series of alpha energy spectrums were obtained under the exposure of a $0.8 \mu \mathrm{Ci}{ }^{241} \mathrm{Am}$ source for $900 \mathrm{~s}$ at different biases. A voltage-dependent alpha spectral peak with a voltage bias changing from $180 \mathrm{~V}$ to $550 \mathrm{~V}$ can be clearly observed, as shown in Fig. 3b. From the Hecht equation of single-polarity charge transport, the calculated hole mobilitylifetime $\mu \tau$ product could be $(0.4-1.6) \times 10^{-3} \mathrm{~cm}^{2} \mathrm{~V}^{-1}$ (as shown in Fig. 3c). However, the relatively high device dark current (200 nA) apparently limits the device performance. To this end, He et al. firstly reported the alpha particle detector based on an all-inorganic perovskite $\mathrm{CsPbBr}_{3}$ with an asymmetric In/ $\mathrm{CsPbBr}_{3} / \mathrm{Au}$ structure. ${ }^{91}$ The crystal was grown by the Bridgman method and cut into $3 \mathrm{~mm} \times 3 \mathrm{~mm} \times 1 \mathrm{~mm}$ for device fabrication. Due to the large difference in work functions between the In $\left(\phi_{\mathrm{In}}=4.1 \mathrm{eV}\right)$ and $\mathrm{Au}\left(\phi_{\mathrm{Au}}=5.1 \mathrm{eV}\right)$ electrodes, the large potential barriers for both hole and electron can be formed on the In and Au sides, respectively, which further suppressed the dark current of the device under reverse bias. As shown in Fig. 3d and e, a low dark current of $2 \mathrm{nA} \mathrm{cm}^{-2}$ was obtained at $-1 \mathrm{~V}$, and it could be increased to $100 \mathrm{nA} \mathrm{cm} \mathrm{cm}^{-2}$ at a bias of $-100 \mathrm{~V}$; while a large dark current of $300 \mathrm{nA} \mathrm{cm}^{-2}$ at a $100 \mathrm{~V}$ forward bias was obtained. A voltage-dependent alpha spectral peak was also shown in all-inorganic perovskite alpha radiation detector (as shown in Fig. 3f), with a peak resolution of $15 \%$. Notably, this alpha particle detector can be operated at a low bias of $-6 \mathrm{~V}$ compared to other perovskite-based alpha particle detectors which usually require large operation voltages. 
a
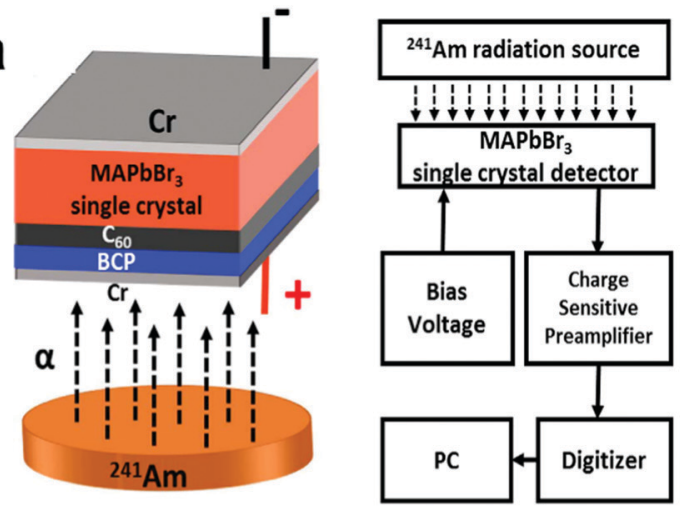

C

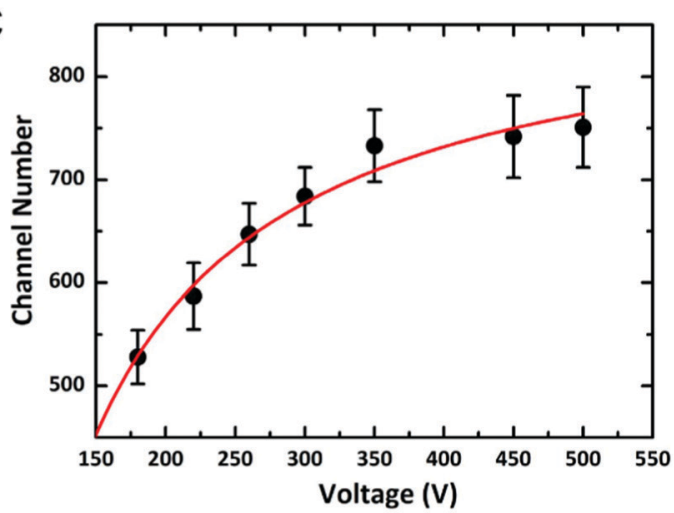

d

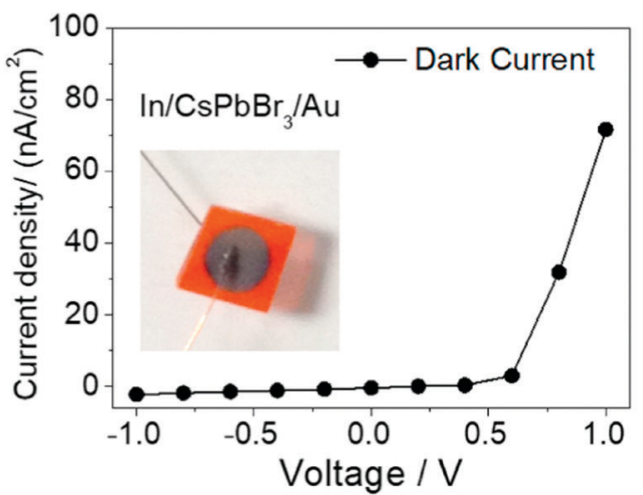

e

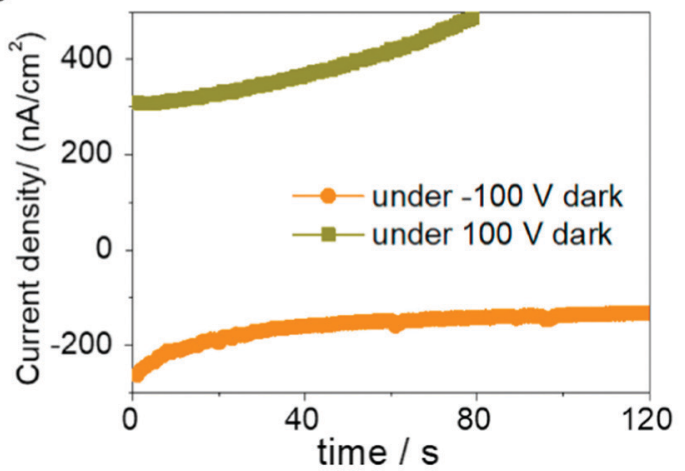

b

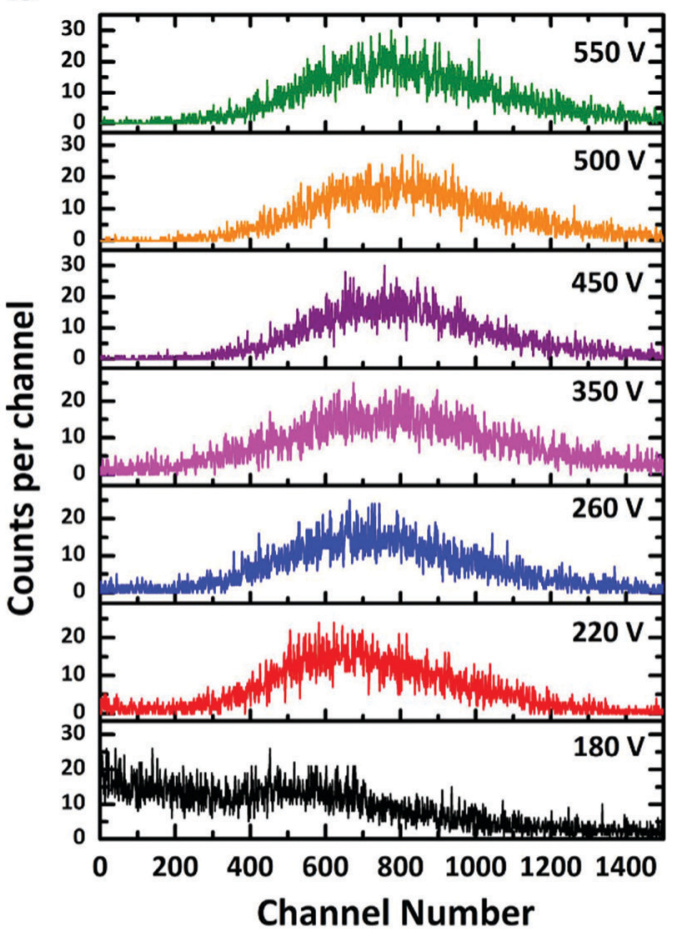

f

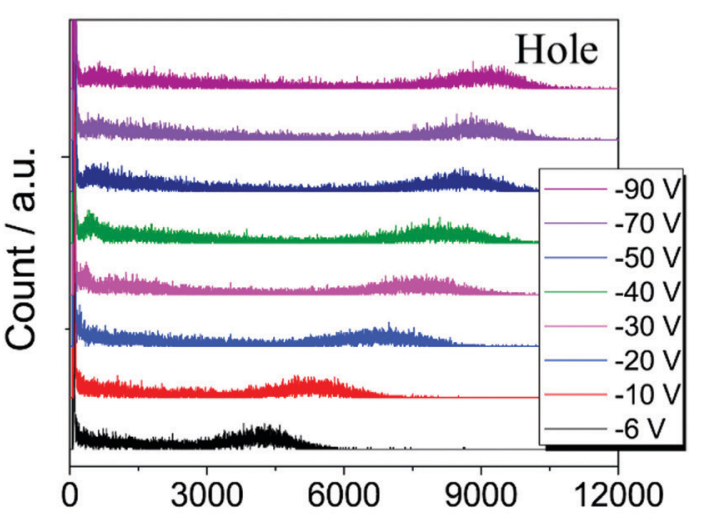

g

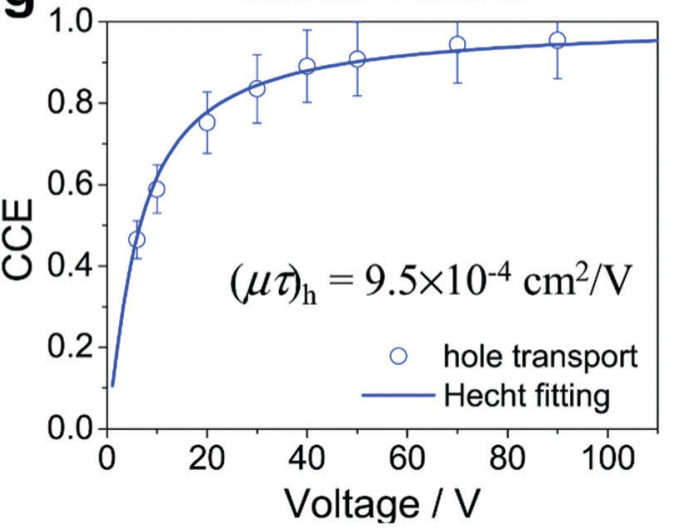

Fig. 3 (a) Schematic illustration of the alpha particle detector based on $\mathrm{MAPbBr}_{3}$ single crystal with the size of $5 \times 5 \times 2 \mathrm{~mm}^{3}$. (b) Voltage-dependent alpha spectra obtained from $\mathrm{MAPbBr}_{3}$ single crystal detector. (c) Spectral peak centroids at different voltage biases. Black points represent experimental data, with a red line fitted from the Hecht equation. Reproduced with permission. ${ }^{101}$ Copyright 2017 , Elsevier. (d) $I-V$ curve characterization of In/CsPbBr $3 / \mathrm{Au}$ alpha particle detector with the structure of $\mathrm{In} / \mathrm{CsPbBr}_{3}\left(3 \times 3 \times 1 \mathrm{~mm}^{3}\right) / \mathrm{Au}$ under the dark condition at bias from $-1 \mathrm{~V}$ to $1 \mathrm{~V}$. (e) Temporal I-V characterization of the $\mathrm{In} / \mathrm{CsPbBr}_{3} / \mathrm{Au}$ detector under forward and reverse applied bias conditions. (f) Voltage-dependent alpha spectra were obtained under different reverse biases. (g) Mobility-lifetime product calculation for hole obtained by fitting the Hecht equation. Reproduced with permission. ${ }^{91}$ Copyright 2019 , Elsevier. 
The hole mobility-lifetime product of $9.4 \times 10^{-4} \mathrm{~cm}^{2} \mathrm{~V}^{-1}$ was obtained from fitting the Hecht equation (Fig. 3g), which exhibits the competitive device performance of all-inorganic perovskites working for alpha particle detectors.

Besides direct alpha particle detectors, lead-based halide perovskites can also be suitable for fabricating indirect scintillators for detecting alpha particles. The most outstanding merit of halide perovskite-based scintillators is their fast response speed, which usually lies in the magnitude of nanosecond. Mykhaylyk et al. reported high light-yield (LY) alpha particle scintillators based on hybrid perovskite $\mathrm{MAPbBr}_{3}$ nanocrystals with an ultrafast response time at low temperature. ${ }^{84}$ As depicted in Fig. 4a, hybrid perovskite $\mathrm{MAPbBr}_{3}$ shows an increasing light output yield with decreasing temperature, which is much higher than those of the commercial LYSO-Ce-based scintillators at around $150 \mathrm{~K}$ and CsI-based scintillators at around $50 \mathrm{~K}$. Moreover, hybrid perovskite $\mathrm{MAPbBr}_{3}$ also exhibited a rapid and intense alpha response with fast and slow decay times of $0.1 \mathrm{~ns}$ and $1 \mathrm{~ns}$, respectively, at $77 \mathrm{~K}$ compared to the relatively longer decay of $1 \mu$ s for LYSO-Ce (Fig. 4b). Fig. 4c shows the pulse height spectra of the scintillators based on $\mathrm{MAPbBr}_{3}$ and CsI with a distinct Gaussian shape peak of $5.5 \mathrm{MeV}$ alpha particle from ${ }^{241} \mathrm{Am}$ source, which exhibited the comparable alpha detecting ability of $\mathrm{MAPbBr}_{3}$ with that of the commercial scintillators.

Currently, lithium-doped 2D lead halide perovskite $(\mathrm{PEA})_{2} \mathrm{PbBr}_{4}$ is also being used for multiple radiation detectors
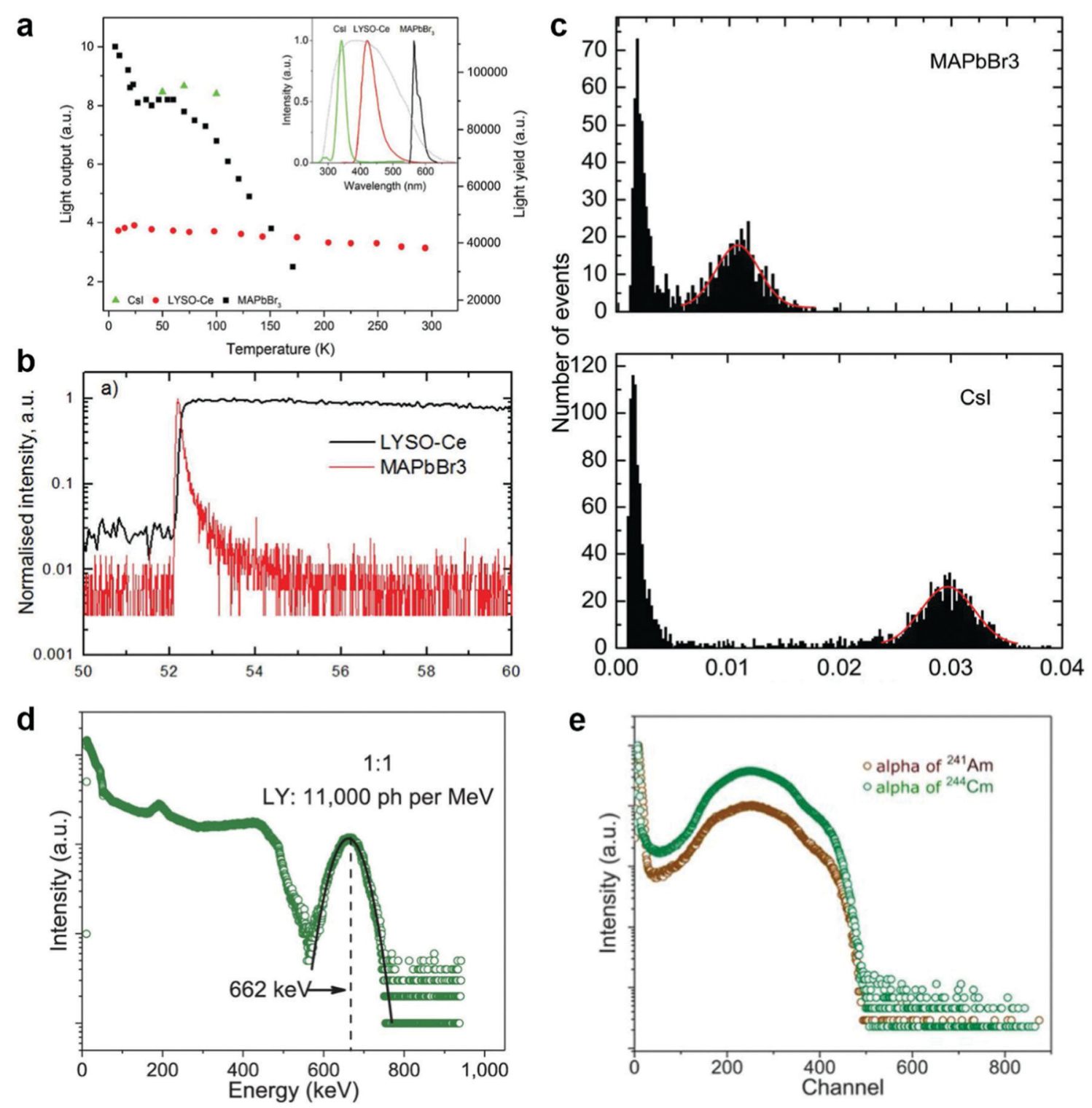

Fig. 4 (a) Scintillator light output measurement as a function of temperature for $\mathrm{MAPbBr}_{3}$ (black), LYSO-Ce (red), and Csl (green) under ${ }^{241} \mathrm{Am}$ source. (b) Normalised scintillator decay of $\mathrm{MAPbBr}_{3}$ crystal (red) and LYSO-Ce (black). (c) Alpha pulse height spectra of $\mathrm{MAPbBr}_{3}$ and CSI scintillator at $50 \mathrm{~K}$. The pulse height distributions that signify the scintillation response are fitted by Gaussian fitting (red lines). Reproduced with permission. ${ }^{84}$ Copyright 2019, RSC. (d) Pulse height spectra of Li-doped (PEA) ${ }_{2} \mathrm{PbBr}_{4}$ with Gaussian fitting to extract light yield. (e) Alpha pulse height spectra of (PEA) $\mathrm{PbBr}_{4}$ scintillator. Reproduced with permission. ${ }^{102}$ Copyright 2020, Nature publishing group. 
and scintillators, which was reported by Xie et al. for the first time. ${ }^{102}$ With a lithium dopant, light yield of $(\mathrm{PEA})_{2} \mathrm{PbBr}_{4}$-based devices could be increased up to $11000 \mathrm{ph} \mathrm{MeV}^{-1}$, and a fast decay time of 11 ns was also obtained (in Fig. 4d). Fig. 4e displays the pulse height spectra results of the (PEA) ${ }_{2} \mathrm{PbBr}_{4}$ scintillator under radiation from ${ }^{241} \mathrm{Am}$ and ${ }^{224} \mathrm{Cm}$ sources. Table 2 summarizes the figures of merit of halide perovskitebased alpha detectors. Compared to the commercial alpha particle detectors and scintillators which require high-cost production lines, lead-based halide perovskites, taking the obvious advantages of low cost and multiply functional detectability, have already shown impressive potential for next-generation alpha particle detection applications.

\subsection{Beta particle detectors}

Holding a moderate penetration ability, beta-rays, usually consisting of electrons or positrons, play an important role in surface radiative contamination surveillance ${ }^{103}$ and cancer cell treatment. ${ }^{104}$ Beta particles can interact with atoms by elastic scattering and inelastic scattering. As mentioned above, elastic scattering happens when beta particles are scattered with nuclei which only changes the trajectory and inelastic scattering happens between beta particles and electrons in atoms which produces other electrons or photon signals. The ratio between elastic scatter and inelastic scatter $\eta$ is higher for the element with a larger $Z$, which means that more inelastic scatterings happen in the low- $Z$ elements and thus more photons are emitted and high scintillator performance can be obtained. Recently, beta-ray scintillators were mainly fabricated using organic single crystals, liquids, or plastics, which usually suffer from high production cost, carcinogenicity, complex fabrication, and poor thermal stability. ${ }^{105,106}$ Currently, Yu et al. reported a new type of beta-ray scintillator based on 2D lead halide perovskite $(\mathrm{A})_{2} \mathrm{PbBr}_{4},{ }^{37}$ in which the organic cations can be butylamine (BA), octylamine (OA), stearamine (STA), or dodecylamine (DA). A series of 2D lead halide perovskites were synthesized through a facile solution process, as shown in Fig. 5a-d. After adding $\mathrm{Mn}^{2+}$ as the emission centre, 2D lead halide perovskites show a significant increase in PL-QY values, as shown in Fig. 5e-h. Fig. 5i displays the working set-up of a beta-ray scintillator based on 2D lead halide perovskite with scintillation signal captured using a CCD camera. Moreover, the resultant beta particle scintillator exhibited a low detection limit of $0.1 \mathrm{mCi}$ (Fig. $5 \mathrm{j}$ and $\mathrm{k}$ ) and a high decomposition temperature of $300{ }^{\circ} \mathrm{C}$ compared to that in the range of $70-105{ }^{\circ} \mathrm{C}$ for the commercial plastic beta-ray scintillators. Taking the combined advantages of the organic cations for betaparticle detection and the perovskite structure for high PL-QY

Table 2 Figures of merit of the alpha detectors

\begin{tabular}{lllll}
\hline Material & $\begin{array}{l}\mu \tau \text { product } \\
\left(\mathrm{cm}^{2} \mathrm{~V}^{-1}\right)\end{array}$ & $\begin{array}{l}\text { Light yield } \\
(\mathrm{ph} \mathrm{MeV})\end{array}$ & $\begin{array}{l}\text { Response } \\
\text { time }(\mathrm{ns})\end{array}$ & Ref. \\
\hline $\mathrm{MAPbBr}_{3}$ single crystal & $1.6 \times 10^{-3}$ & - & - & 101 \\
$\mathrm{CsPBBr}_{3}$ single crystal & $9.4 \times 10^{-4}$ & - & - & 91 \\
$\mathrm{MAPbBr}_{3}$ nanoparticle & - & 90000 & $0.1 / 1$ & 84 \\
$(\mathrm{PEA})_{2} \mathrm{PbBr}_{4}$ single crystal & - & 11000 & 11 & 102
\end{tabular}

values, hybrid 2D lead halide perovskites shed light on highperformance beta-ray detectors.

\section{Photon-radiation detection by lead halide perovskites}

\subsection{Gamma-ray detectors}

Gamma-rays, as a form of electromagnetic waves, have the shortest wavelength and the highest energy, and are usually emitted from atomic nuclei. Gamma-rays can also be detected using an indirect scintillator or direct photodetector that records a gamma-ray spectrum through the voltage mode. So, the most important figure of merit of a gamma-ray detector is gamma energy resolution, which can be defined as the ratio between the FWHM of the gamma-ray peak and the gamma-ray's energy. Similar to the other photons, gamma-rays show an exponential decay in their intensity when they pass through objects. The gamma-ray intensity can be given by

$$
I=I_{0} \mathrm{e}^{-\mu x}
$$

where $x$ is the thickness of the active material and $\mu$ is the absorption coefficient related to atomic density (atomic number $Z$ ). Due to the high energy and penetration of gamma-rays, several severe requirements should be fulfilled for detecting gamma-rays with high efficiencies, such as a large average atomic number $Z$ for a high stopping power, a large carrier-lifetime product for charge collection, high bulk resistance for low dark current, and superb stability under long time radiation. Nowadays, the state-of-the-art CZT gamma-ray detectors can offer a gamma-ray resolution of $0.5 \%$ at room temperature, ${ }^{107}$ and the high-purity Ge (HPGe) gamma-ray detector holds the highest resolution of $0.3 \%$. However, as mentioned before, these commonly-used gamma-ray detectors usually need to be operated under cryogenic conditions, aiming to suppress the high dark current. ${ }^{108}$

Compared with the halide perovskite polycrystalline films which suffer from high trap density and defects induced from grain boundaries, halide perovskite single crystals with appropriate thickness and low trap density have gained favors for gamma-ray detectors. Yakunin et al. reported the fabrication of the gamma-ray detectors based on $\mathrm{FAPbI}_{3}$ single crystals at room temperature for the first time. ${ }^{109}$ In this work, two largesized (3-12 mm) hybrid perovskite $\mathrm{MAPbI}_{3}$ and $\mathrm{FAPbI}_{3}$ single crystals were grown through a facile solution-processed method. Fig. 6a shows the photocurrent measured as a function of voltage bias under the radiation of $\mathrm{Cu} \mathrm{K} \alpha \mathrm{X}$-ray. By fitting the data using the Hecht equation, perovskite $\mathrm{MAPbI}_{3}$ exhibited a high $\mu \tau$ product of around $10^{-2} \mathrm{~cm}^{2} \mathrm{~V}^{-1}$. The fabricated gamma-ray detector based on $\mathrm{FAPbI}_{3}$ also showed a good response under radiation from ${ }^{241} \mathrm{Am}$, as shown in Fig. 6b. However, the FWHM of the gamma-ray detectors was only $35 \%$, which was limited by the large dark current and poor phase stability of the $\mathrm{FAPbI}_{3}$ single crystals at room temperature. Then, He et al. also demonstrated a well-resolved gamma-ray spectrum via using the $\mathrm{MAPbI}_{3}$ single crystals. ${ }^{110}$ Constructed by 

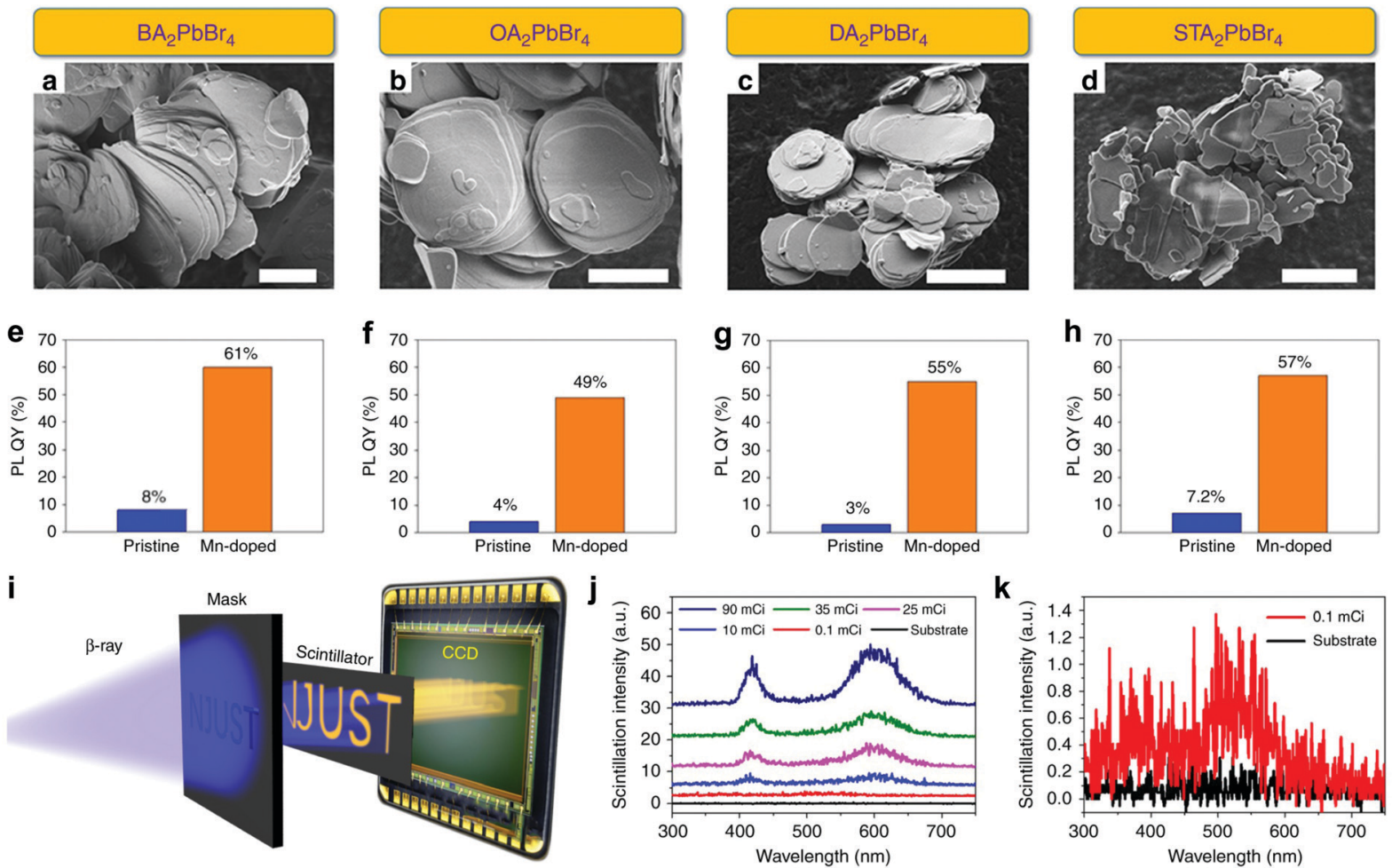

Fig. 5 (a)-(d) $\mathrm{SEM}$ images of $2 \mathrm{D}$ lead halide perovskites $\mathrm{BA}_{2} \mathrm{PbBr}_{4}, \mathrm{OA}_{2} \mathrm{PbBr}_{4}, \mathrm{DA}_{2} \mathrm{PbBr}_{4}$, and $\mathrm{STA}_{2} \mathrm{PbBr}_{4}$. (e)-(h) PL QY measurement before and after $\mathrm{Mn}^{2+}$ addition, with an obvious increase. (i) Set-up for 2D perovskite-based beta-ray scintillator. (j) Scintillation spectra of 2D perovskites under different beta-ray radiation intensities. (k) Scintillation spectra at a beta-ray radiation intensity of $0.1 \mathrm{mCi}$. Reproduced with permission ${ }^{37}$ Copyright 2019 , Nature Publishing Group.

an asymmetric $\mathrm{Ga} / \mathrm{MAPbI}_{3} / \mathrm{Au}$ structure, the dark current was successfully suppressed to less than $100 \mathrm{nA}$ at a $100 \mathrm{~V}$ reverse bias (Fig. 6c). Fig. 6d depicts the gamma-ray spectrum measured using a $\mathrm{Ga} / \mathrm{MAPbI}_{3} / \mathrm{Au}$ photodetector, where a superb peak resolution of $6.8 \%$ was achieved under the radiation of $122 \mathrm{keV}$ of ${ }^{57} \mathrm{Co}$ source. However, the relatively low bandgap and bad thermal stability of $\mathrm{MAPbI}_{3}$ single crystals set a huge limit for their usage in high-energy gamma-ray detection applications. Compared to $\mathrm{MAPbI}_{3}$, both $\mathrm{MAPbCl}_{3}$ and $\mathrm{MAPbBr}_{3}$ are more thermally stable under radiation conditions and can also be synthesized via a solution process. Wei et al. reported a high-performance gamma-ray detector by further modifying the halide component ratio in $\mathrm{MAPbBr}_{x} \mathrm{Cl}_{3-x}$ single crystals. ${ }^{89}$ As the halide component varied from totally $\mathrm{Br}$ to $\mathrm{Cl}$, hybrid perovskite single crystals showed a clear transition from the p-type transport to the n-type behaviour. Particularly, it is found that the mixed hybrid perovskite $\mathrm{MAPbBr}_{2.94} \mathrm{Cl}_{0.06}$ single crystal was almost intrinsic, thus leading to a ten-fold improved bulk resistivity of $3.6 \times 10^{9} \Omega \mathrm{cm}$. The dopant technique also offered an increased $\mu \tau$ product of $1.8 \times 10^{-2} \mathrm{~cm}^{2} \mathrm{~V}^{-1}$. Besides crystal quality improvement via the dopant strategy, efficient device designs also made a great contribution to the radiation detecting performance. As shown in Fig. 6e, the surface/edge leakage current was mitigated by a guard ring electrode. Being capable of detecting higher-energy gamma-rays, the $\mathrm{MAPbBr}_{2.94} \mathrm{Cl}_{0.06}$ photodetector exhibited a resolution of $6.5 \%$ under $665 \mathrm{keV}$ gamma-ray radiation from a ${ }^{137} \mathrm{Cs}$ source (Fig. 6f).
Besides hybrid lead halide perovskites, all-inorganic lead halide perovskites, in which organic cations are usually replaced by inorganic cation $\mathrm{Cs}^{+}$, also hold huge potential for gamma-ray detection. All-inorganic perovskite $\mathrm{CsPbBr}_{3}$ has a high average atomic number of 65.9 which is much higher than that of CZT (50.2), thus leading to a larger attenuation coefficient and a higher stopping power. He et al. have successfully obtained a high-resolution gamma-ray spectrum by using $\mathrm{CsPbBr}_{3}$ crystals. $^{35}$ Grown using the Bridgman technique, perovskite $\mathrm{CsPbBr}_{3}$ single crystal can be easily diced into different shapes (Fig. 7a). Asymmetric device structure with $\mathrm{CsPbBr}_{3}$ single crystal working as the active layer was also applied in this work with $\mathrm{Ga}$ and Au used as electrodes. As mentioned above, because of the different Work Functions between the Ga and Au electrodes, the dark current was highly suppressed even under high reverse bias. Fig. $7 \mathrm{~b}$ and $\mathrm{c}$ show the dark current characterization of the asymmetrically-structured $\mathrm{Ga} / \mathrm{CsPbBr}_{3} / \mathrm{Au}$ and the symmetricallystructured $\mathrm{Au} / \mathrm{CsPbBr}_{3} / \mathrm{Au}$ devices, respectively, where the asymmetric devices displayed a $10^{4}$-times lower dark current at $-200 \mathrm{~V}$ bias. Noticeably, $\mathrm{CsPbBr}_{3}$ also maintained a good $\mu \tau$ product of $1.34 \times 10^{-3} \mathrm{~cm}^{2} \mathrm{~V}^{-1}$. Furthermore, when exposed to a $5 \mu \mathrm{Ci}{ }^{137} \mathrm{Cs}$ source, the asymmetric photodetector achieved a peak resolution of $3.8 \%$ at $662 \mathrm{keV}$ (Fig. 7d). Recently, He et al. also reported a gamma-ray photodetector with an asymmetric In/CsPbBr 3 /Au structure, ${ }^{91}$ which was capable of detecting both alpha-particles and gamma-rays. Sharing a similar principle, a $4.8 \%$ photopeak resolution under the radiation of ${ }^{57} \mathrm{Co}$ source at 


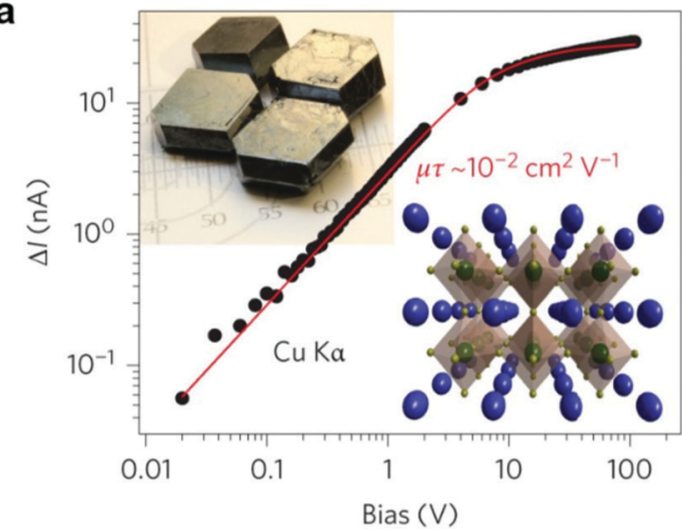

C
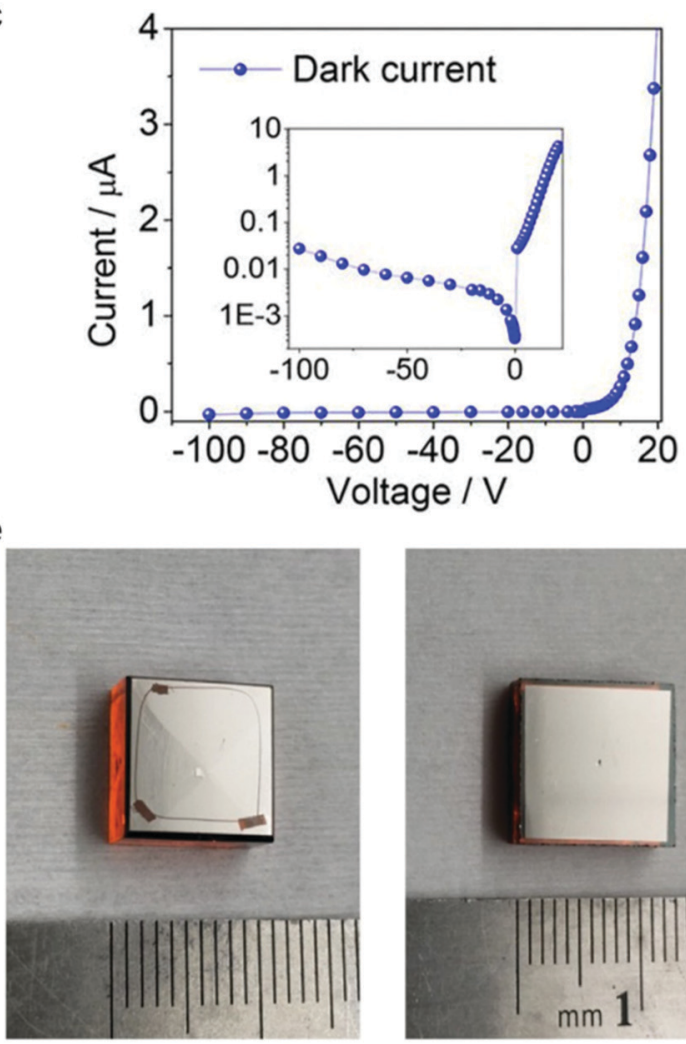

b

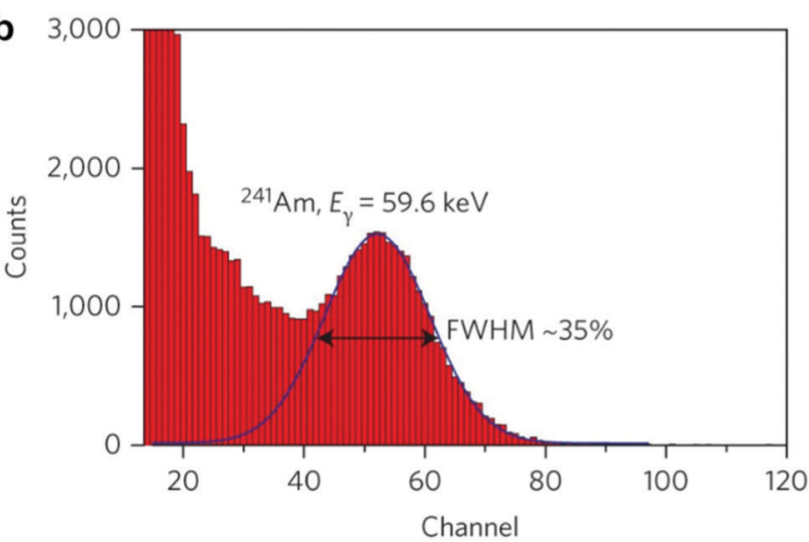

d
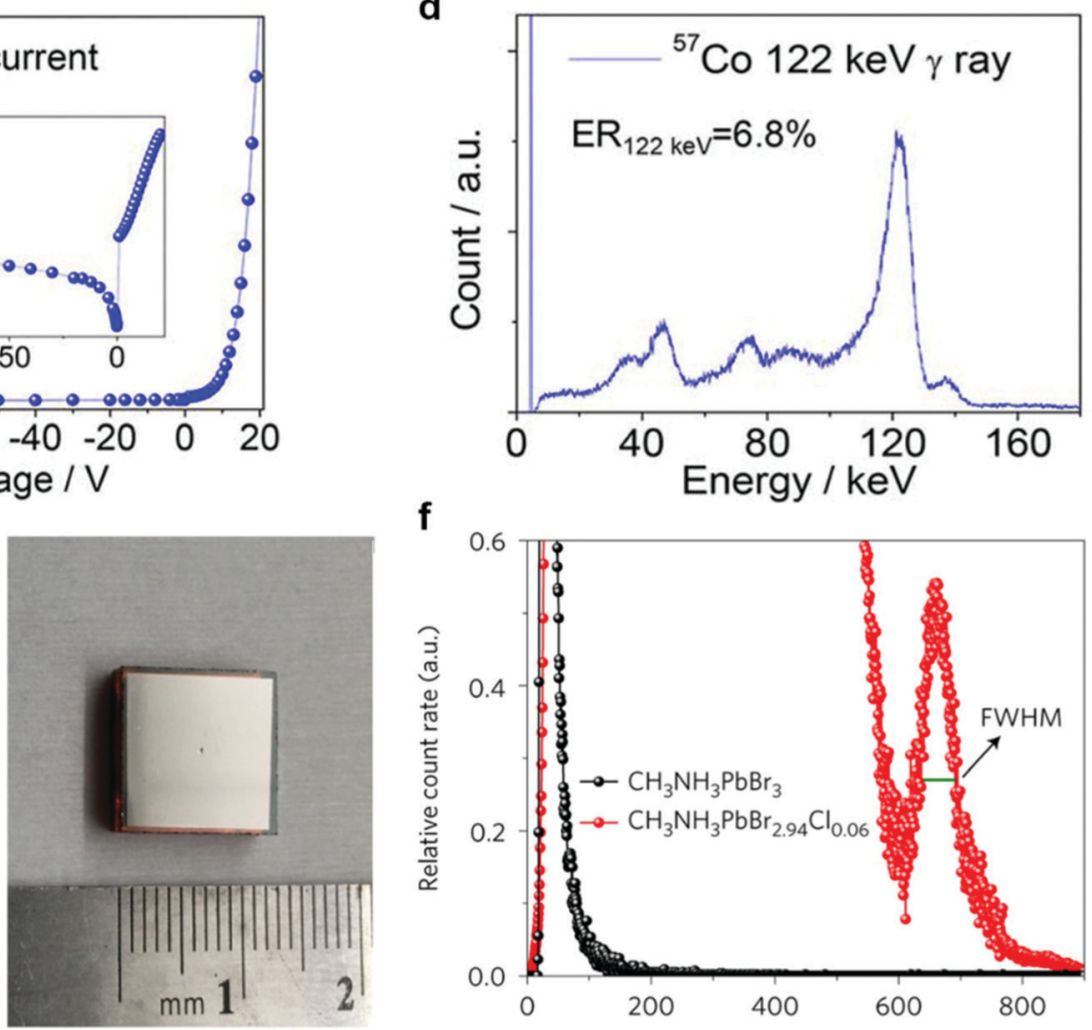

f

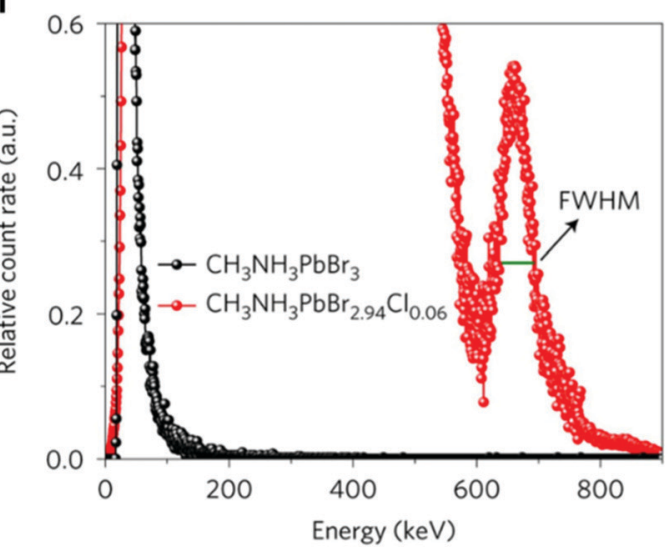

Fig. 6 (a) Bias dependency of photocurrent in $\mathrm{MAPb}_{3}$ single crystals generated by $\mathrm{Cu} \mathrm{K} \alpha \mathrm{X}$-rays at $8 \mathrm{KeV}$. The red line indicates a fitting from the Hecht equation with $\mu \tau$ of $10^{-2} \mathrm{~cm}^{2} \mathrm{~V}^{-1}$. (b) Energy resolved spectrum of ${ }^{241} \mathrm{Am}$ by FAPbl 3 single crystal. Reproduced with permission. ${ }^{109} \mathrm{Copyright} 2016$, Nature Publishing Group. (c) I-V curve of Ga/MAPb| $/$ /Au photodetector under dark conditions. (d) Energy resolved spectrum of ${ }^{57} \mathrm{Co}$ by $\mathrm{MAPb} / 3$ single $\mathrm{crystals}$. Reproduced with permission. ${ }^{110}$ Copyright 2017, ACS. (e) Left: Top view of the guard ring electrode side of $\mathrm{MAPbBr}_{2.94} \mathrm{Cl}_{0.06}$ photodetector. Right: Top view of the anode electrode side. (f) Enlarged image of the photopeak region from ${ }^{137} \mathrm{Cs}$ spectrum obtained using $\mathrm{MAPbBr}_{2.94} \mathrm{Cl}_{0.06}$ photodetector (red) and $\mathrm{MAPbBr}_{3}$ photodetector. Reproduced with permission. ${ }^{89}$ Copyright 2017, Nature Publishing Group.

$122 \mathrm{keV}$ was achieved. Lately, He et al. further improved the device structure via using a low work-function metal in the eutectic Ga-In alloy (EGaIn), ${ }^{111}$ which enabled a record-high gamma-ray peak resolution of $1.4 \%$ by the $\mathrm{EGaIn} / \mathrm{CsPbBr}_{3} / \mathrm{Au}$ gamma-ray detector with a planar structure (Fig. 7e). However, the planarstructured radiation detector suffers from a decrease in peak resolution when enlarging the crystal size. With a hemispherical device structure as shown in Fig. 7f, a unipolar radiation device with electrons being screened was obtained, and a larger-sized
$\mathrm{CsPbBr}_{3}$ crystal can be applied into this gamma-ray detector with a high peak-resolution of $1.8 \%$.

Lead-based halide perovskites have also been used in indirect gamma-ray scintillators, owing to their high photoluminescence (PL) and light-emitting properties. Recently, Xu et al. reported a gamma-ray scintillator based on hybrid perovskite $\mathrm{MAPbBr}_{0.05^{-}}$ $\mathrm{Cl}_{2.95}$ single crystal. ${ }^{112}$ In this work, a series of $\mathrm{MAPbBr}_{x} \mathrm{Cl}_{3-x}$ single crystals were grown via a solution-processed method, and then the obtained single crystals were further integrated 
a

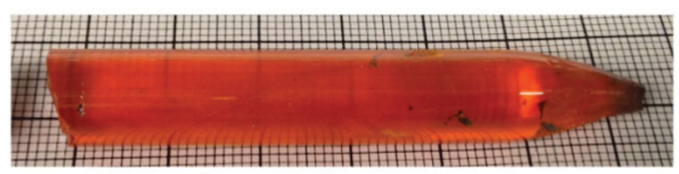

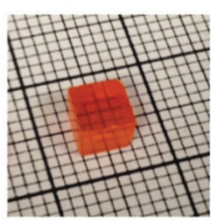

$4 \times 4 \times 3 \mathrm{~mm}^{3}$

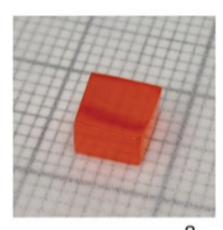

$5 \times 5 \times 3 \mathrm{~mm}^{3}$

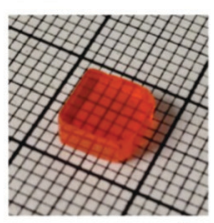

$6 \times 6 \times 3 \mathrm{~mm}^{3}$

C

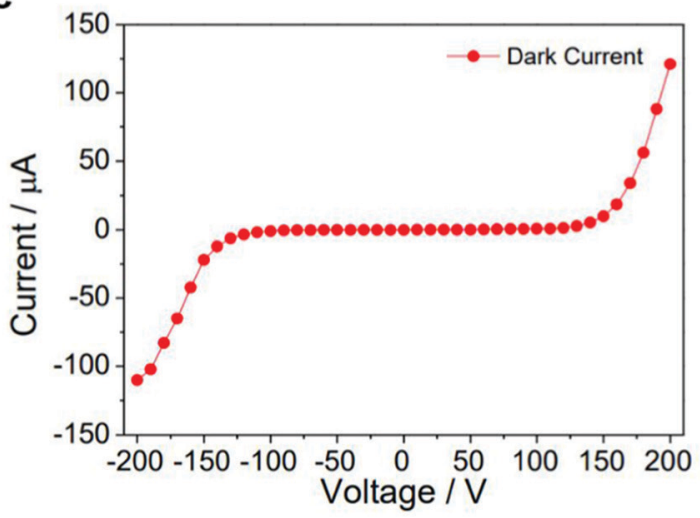

b

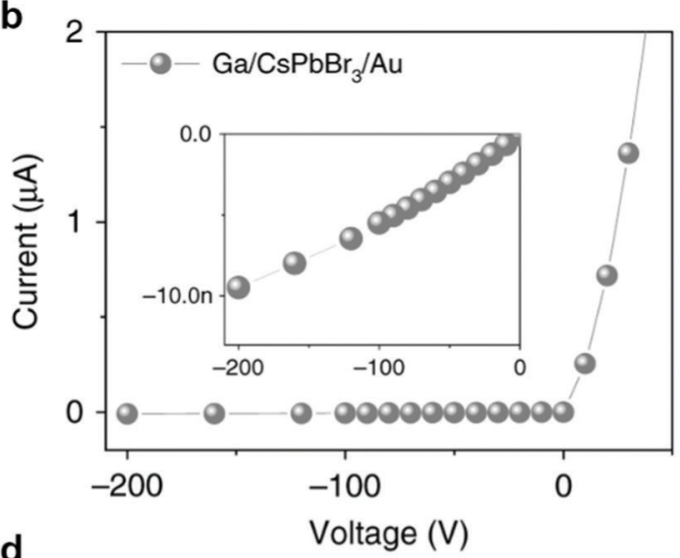

d

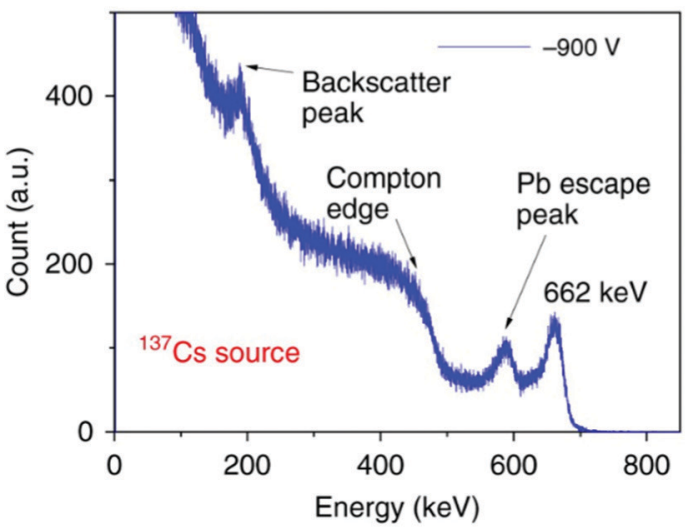

e

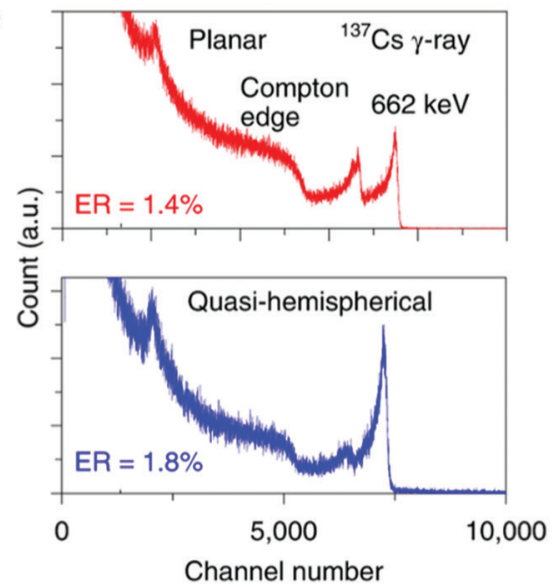

f

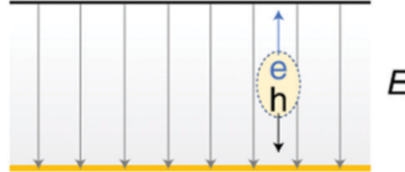

Planar type

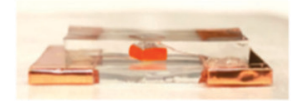

$\sim 2.5 \times 2 \times 1.33 \mathrm{~mm}^{3}$
E

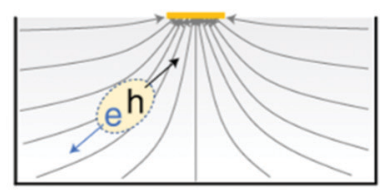

Quasi-hemispherical type

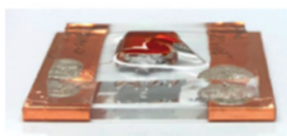

$6 \times 6 \times 3.02 \mathrm{~mm}^{3}$

Fig. 7 (a) $\mathrm{CsPbBr}_{3}$ single crystal grown using the Bridgman technique. (b) I-V curve of the $\mathrm{Ga} / \mathrm{CsPbBr} / \mathrm{Au}$ asymmetric device. (c) I-V curve of the Au/ $\mathrm{CsPbBr} 3 / \mathrm{Au}$ device, with a large dark current at $-200 \mathrm{~V}$. (d) Gamma spectrum obtained by $\mathrm{CsPbBr}_{3}$ single crystal under radiation from ${ }^{137} \mathrm{Cs}$ source. Reproduced with permission. ${ }^{35}$ Copyright 2018, Nature Publishing Group. (e) Gamma spectrum obtained by $\mathrm{CsPbBr}_{3}$ single crystals with different device structures under radiation from a ${ }^{137} \mathrm{Cs}$ source. (f) Schematic illustration of the device operation principle of planar and hemispherical gamma-ray detectors and the corresponding device pictures. Reproduced with permission. ${ }^{111}$ Copyright 2020, Nature Publishing Group.

onto the silicon photomultiplier (SiPM) window as the scintillators. Fig. $8 \mathrm{a}$ and $\mathrm{c}$ show the PL spectrum of $\mathrm{MAPbBr}_{x} \mathrm{Cl}_{3-x}$ single crystals by tuning the ratio of $\mathrm{Br}$ and $\mathrm{Cl}$ components. A nearband-edge emission was obtained for $\mathrm{MAPbBr}_{0.05} \mathrm{Cl}_{2.95}$ single crystal. The pulse height spectra of $1.7 \mu \mathrm{Ci}{ }^{137} \mathrm{Cs}$ source were successfully acquired at room temperature (as shown in Fig. 8b). Furthermore, 0D all-inorganic halide perovskitebased gamma-ray scintillators were also fabricated by $\mathrm{Xu}$ and his colleagues. ${ }^{90} \quad 0 \mathrm{D} \quad \mathrm{Cs}_{4} \mathrm{PbBr}_{6}$ embedded with $\mathrm{CsPbBr}_{3}$ nanocrystals was synthesized by a facile solution method. The obtained $0 \mathrm{D} \mathrm{Cs}_{4} \mathrm{PbBr}_{6} / \mathrm{CsPbBr}_{3}$ nanocrystal composites were further coated onto a photomultiplier tube (PMT) for fabricating gamma-ray scintillators, as shown in Fig. 8d. Fig. 8e exhibits the obtained pulse height spectra under the radiation from ${ }^{241} \mathrm{Am}$, where a high resolution of $3.0 \%$ was achieved by the $0 \mathrm{D}$ $\mathrm{Cs}_{4} \mathrm{PbBr}_{6} / \mathrm{CsPbBr}_{3}$-based scintillator compared with that of the commercial $\mathrm{NaI}(\mathrm{Tl}$ ) (resolution of $17.8 \%$ ). Moreover, a fast decay time of $10 \mathrm{~ns}$ and a high light yield of $64000 \mathrm{ph} \mathrm{MeV}^{-1}$ were 
a

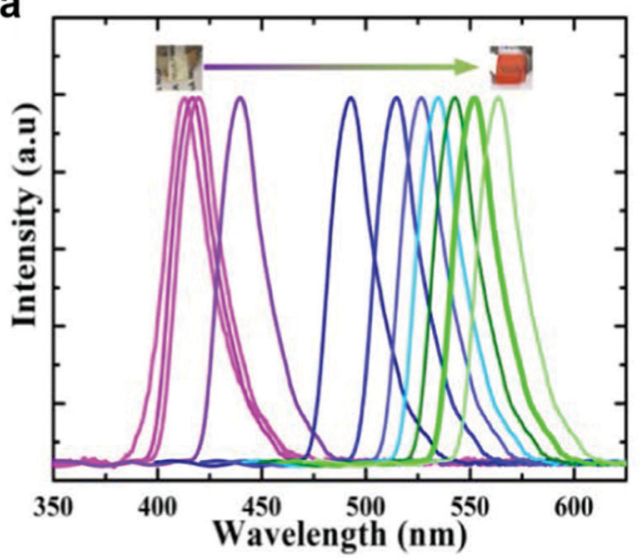

C
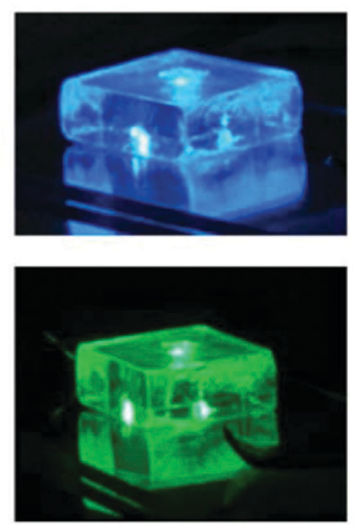

d

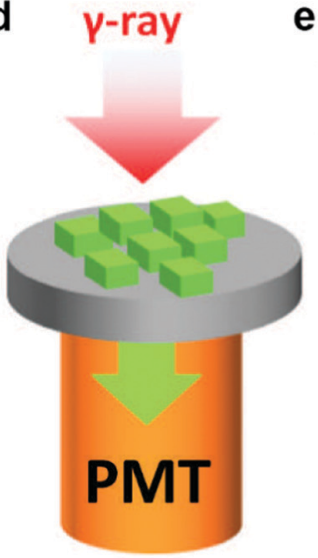

e b

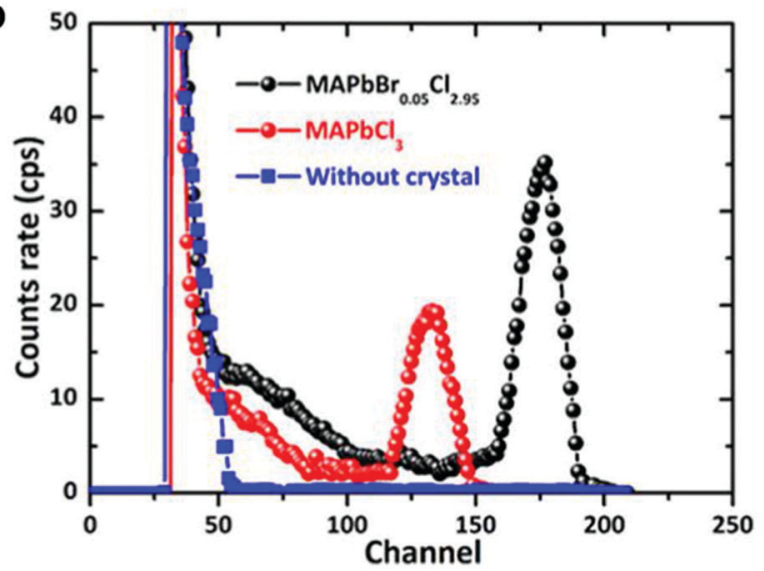

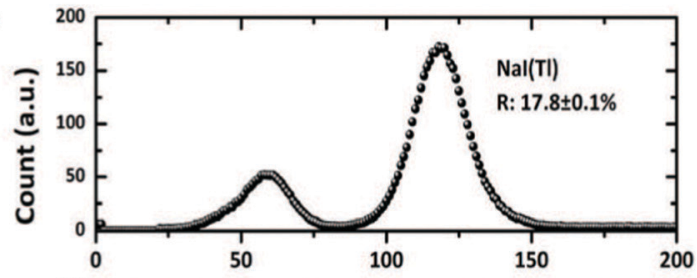

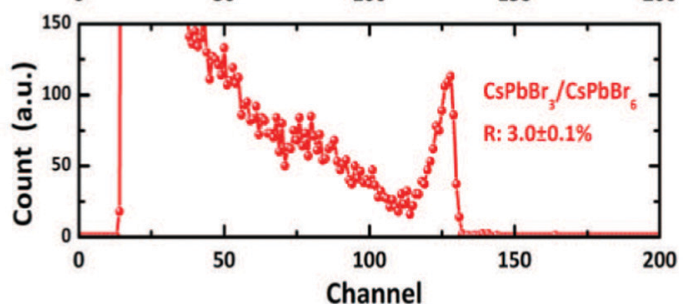

Fig. 8 (a) X-Ray induced photoluminescence of $\mathrm{MAPbBr}_{x} \mathrm{Cl}_{3-x}$ single crystals. (b) Pulse height spectra acquired by $\mathrm{MAPbCl}_{3}$ and $\mathrm{MAPbBr}_{0.05} \mathrm{Cl}_{2.95}$ under the radiation of a $1.4 \mu \mathrm{Ci}^{137} \mathrm{Cs}$ source. Reproduced with permission. ${ }^{112}$ Copyright 2019, ACS. (c) Optical images of $\mathrm{MAPbBr}_{x} \mathrm{Cl}_{3-x}$ single crystals excited by a $365 \mathrm{~nm}$ laser. (d) Schematic of gamma-ray scintillator set up. (e) Pulse height spectra acquired by the commercial $\mathrm{NaT}\left(\mathrm{Tl}_{1}\right)$ and $\mathrm{CsPbBr}_{3} / \mathrm{Cs}_{4} \mathrm{PbBr}_{6}$ scintillator under the radiation of ${ }^{241} \mathrm{Am}$ source. Reproduced with permission. ${ }^{90}$ Copyright 2020, RSC.

obtained as well. Besides the 3D- and 0D-formed halide perovskites (like $\mathrm{CsPbBr}_{3}$ crystals and $0 \mathrm{D} \mathrm{Cs}_{4} \mathrm{PbBr}_{6}$ materials embedded with $\mathrm{CsPbBr}_{3}$ nanocrystals), layered halide perovskite $\left(\mathrm{C}_{6} \mathrm{H}_{5} \mathrm{C}_{2} \mathrm{H}_{4} \mathrm{NH}_{3}\right)_{2} \mathrm{PbBr}_{4}$ has also shown good scintillation when excited by gamma-rays. ${ }^{113}$ In the work, the large-sized $(27 \times 13 \times$ $4 \mathrm{~mm}$ ) layered perovskite crystals were synthesized by a solutionprocessed method. The bulk crystal sample showed a high light yield of $14000 \mathrm{ph} \mathrm{MeV}^{-1}$ and a fast decay time of 11 ns. Table 3 summarizes the figures of merit of the lead halide perovskitebased gamma-ray detectors and other commercial gamma-ray detectors based on other materials. Even though halide perovskite-based gamma-ray detectors have a relatively lower resolution (about 1\%) as compared with the state-of-the-art CZT gamma-ray detector, its low-temperature growth method and reasonable production cost indisputably blaze a new way for new generation gamma-ray detectors which are capable of both direct and indirect detection applications.

\subsection{X-Ray detectors}

$\mathrm{X}$-Rays, as a kind of electromagnetic wave with high penetration, is one of the most widely-used forms of radiation in modern society. Since their discovery by Rontgen in 1895, X-rays were immediately applied into the field of medical applications due to their high sensitivity to different materials and thus enabled high-resolution imaging applications. ${ }^{115}$ In the 20th century, $\mathrm{X}$-rays have shown their ability in the areas of crystallography, imaging, microscopy, and even been used in World War I. Nowadays, the application of X-rays covers more broad fields, including medicine, food safety, environmental surveillance, industry, science research studies, etc. ${ }^{2,6,116}$ Similar to gamma-rays, $\mathrm{X}$-rays can be detected using either direct photodetectors coupled with semiconductor photodiodes or indirect scintillators. While aiming to get high imaging performance, extra consideration of sensitivity, spatial resolution, and lowest detectable dose rate need to be taken for X-ray detectors.

5.2.1. X-Ray photodetector. As mentioned before, leadbased halide perovskites, which hold a large bulk resistance, strong stopping power, and high mobility-lifetime $\mu \tau$ product, are promising for high-energy photon detection devices, which can be also applied to X-ray detection applications. Recently, Wei et al. reported a highly sensitive X-ray detector based on hybrid perovskite $\mathrm{MAPbBr}_{3}$ single crystal. ${ }^{38}$ A non-stoichiometry precursor ratio with $\mathrm{MABr} / \mathrm{PbBr}_{2}$ of 0.8 was used for the growth of high-quality perovskite single crystals (Fig. 9a), where the 
Table 3 Figures of merit of conventional gamma-ray detectors

\begin{tabular}{|c|c|c|c|c|c|}
\hline Material & $\begin{array}{l}\text { Bandgap } \\
\text { (eV) }\end{array}$ & $\begin{array}{l}\mu \tau \text { product } \\
\left(\mathrm{cm}^{2} \mathrm{~V}^{-1}\right)\end{array}$ & Source & $\begin{array}{l}\text { Energy } \\
\text { resolution (\%) }\end{array}$ & Ref. \\
\hline HPGe & 0.66 & $>1$ & ${ }^{137} \mathrm{Cs}$ & 0.2 & 39 \\
\hline CZT & $1.5-1.6$ & $>10^{-2}$ & ${ }^{137} \mathrm{Cs}$ & 0.5 & 107 \\
\hline $\mathrm{MAPbI}_{3}$ & 1.5 & $0.8 \times 10^{-3}$ & ${ }^{57} \mathrm{Co}$ & 6.8 & 110 \\
\hline $\mathrm{MAPbBr}_{2.94} \mathrm{Cl}_{0.06}$ & 2.2 & $1.8 \times 10^{-2}$ & ${ }^{137} \mathrm{Cs}$ & 6.5 & 89 \\
\hline $\mathrm{CsPbBr}_{3}$ & 2.3 & $1.3 \times 10^{-3}$ & ${ }^{137} \mathrm{Cs}$ & 3.8 & 35 \\
\hline $\mathrm{CsPbBr}_{3}$ & 2.3 & $8 \times 10^{-3}$ & ${ }^{137} \mathrm{Cs}$ & 1.4 & 111 \\
\hline $\mathrm{MAPbBr}_{0.05} \mathrm{Cl}_{2.95}$ & 3.0 & - & ${ }^{137} \mathrm{Cs}$ & 10.5 & 112 \\
\hline $\mathrm{Cs}_{4} \mathrm{PbBr}_{6} / \mathrm{CsPbBr}_{3}$ & - & - & ${ }^{241} \mathrm{Am}$ & 3.0 & 90 \\
\hline $\mathrm{NaI}(\mathrm{Tl})$ & 3.0 & - & ${ }^{137} \mathrm{Cs}$ & 5.6 & 114 \\
\hline
\end{tabular}

obtained $\mathrm{MAPbBr}_{3}$ crystals showed a record $\mu \tau$ product of $1.2 \times$ $10^{-2} \mathrm{~cm}^{2} \mathrm{~V}^{-1}$. As shown in Fig. 9b, the fabricated X-ray detector exhibited a great linear relationship between photocurrent and $\mathrm{X}$-ray dose. The high sensitivity of $80 \mu \mathrm{C} \mathrm{Gy}{ }_{\text {air }}{ }^{-1} \mathrm{~cm}^{-2}$ was achieved, which was ten times higher than that of the CZT detector under the same bias. The lowest detectable X-ray dose rate was observed as $0.5 \mu \mathrm{Gy}_{\text {air }} \mathrm{s}^{-1}$, which is enough to satisfy the medical diagnostics $\left(5.5 \mu \mathrm{Gy}_{\text {air }} \mathrm{S}^{-1}\right)$ requirement. Except for the single-crystal perovskites, $\mathrm{MAPbI}_{3}$ polycrystalline films were also applied in the direct X-ray detectors, ${ }^{117}$ where a high sensitivity of $2527 \mu \mathrm{C} \mathrm{Gy}{ }_{\text {air }}{ }^{-1} \mathrm{~cm}^{-2}$ was achieved. Lately, a quasi-monocrystalline $\mathrm{CsPbBr}_{3}$-based X-ray detector was fabricated by Pan et al. ${ }^{92}$ By using an easy hot-press method, a $\mathrm{CsPbBr}_{3}$ film with a thickness of hundreds of micrometres was obtained, as shown in Fig. 9c. Notably, the X-ray detectors held a record-high sensitivity of $55684 \mu \mathrm{C} \mathrm{Gy}_{\text {air }}{ }^{-1} \mathrm{~cm}^{-2}$ under a $5.0 \mathrm{~V} \mathrm{~mm}^{-1}$ electric field (Fig. 9d). The all-inorganic components enabled excellent film stability with only a slight reduction in sensitivity after exposure to air for 9 months. Gou et al. have also successfully grown high-thickness $\mathrm{CsPbBr}_{3}$ polycrystalline films for X-ray detection applications. ${ }^{118} \mathrm{~A}$ dissolve-recrystallization method was used to improve the film quality, which further enhanced the device performance with a high sensitivity of $470 \mu \mathrm{C} \mathrm{Gy}_{\text {air }}{ }^{-1} \mathrm{~cm}^{-2}$ at zero bias under a low $\mathrm{X}$-ray dose radiation of $0.05 \mu \mathrm{Gy}_{\text {air }} \mathrm{S}^{-1}$. Synthesized by a facile hot-injection solution process, $\mathrm{CsPbBr}_{3}$ quantum dots (QDs) can also be used in the devices that can exhibit promising X-ray response performance. ${ }^{119}$ Moreover, flexible X-ray detectors based on $\mathrm{CsPbBr}_{3}$ QD arrays were also fabricated on a PET substrate using the inkjet printing method (Fig. 9e). This easily printable X-ray detector can detect a low dose of $17.2 \mu \mathrm{Gy}_{\text {air }} \mathrm{s}^{-1}$ with a sensitivity of $1450 \mu \mathrm{C} \mathrm{Gy}_{\mathrm{air}}{ }^{-1} \mathrm{~cm}^{-2}$, as shown in Fig. 9f.

5.2.2. X-Ray imaging or scintillators. The first halide perovskite-based X-ray detector for imaging attempt was reported by Yakunin et al. in 2015. ${ }^{88}$ A hybrid perovskite $\mathrm{MAPbI}_{3}$ polycrystalline film was spray-coated on a glass substrate with $\mathrm{Ti}$ / $\mathrm{Au}$ electrodes for X-ray photodetection applications. By scanning various objects under an X-ray beam in the $x-y$ directions, wellresolved images can be directly obtained by the $\mathrm{MAPbI}_{3}$ photodetector, as shown in Fig. 10a. The superb X-ray response enabled by $\mathrm{MAPbI}_{3}$ further proved its promising potential for the perovskite-based X-ray imaging applications, while the integration of perovskites and other semiconductor arrays is still a large obstacle for direct X-ray imaging. Wei et al. used (3-aminopropyl) triethoxysilane molecule to connect the native oxide $\mathrm{Si}$ and perovskite single crystals by the $\mathrm{NH}_{3} \mathrm{Br}$ terminal of the molecule, ${ }^{120}$ which achieved direct X-ray imaging applications without any destruction of the perovskite layers. As shown in Fig. 10b, a solid connection between the Si substrate and hybrid perovskite $\mathrm{MAPbBr}_{3}$ single crystals was obtained which enables the direct X-ray imaging devices. The dark current was also suppressed due to the molecular interlayer between Si and $\mathrm{MAPbBr}_{3}$, which led to a high sensitivity of $2.1 \times 10^{4} \mu \mathrm{C} \mathrm{Gy}_{\text {air }}{ }^{-1} \mathrm{~cm}^{-2}$ and the lowest detectable dose rate of $36 \mathrm{nGy}_{\text {air }} \mathrm{s}^{-1}$. Clear X-ray imaging devices can be obtained with a spatial resolution of

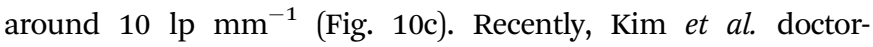
bladed a large-scale MAPbI $_{3}$ film $(10 \times 10 \mathrm{~cm})$ integrated on TFT arrays $^{36}$ (Fig. 10d). A well-resolved X-ray image can be clearly seen with a spatial resolution of $3.1 \mathrm{lp} \mathrm{mm}^{-1}$, as shown in Fig. 10e, while the resolution is still limited by charge diffusion at grain boundaries even when the pixel is only $70 \mu \mathrm{m}$.

Compared to the direct X-ray detector, halide perovskite X-ray scintillators are more suitable for real-time X-ray imaging applications due to their superb light yield and ultrafast photoresponse. As mentioned above, halide perovskite QDs and nanocrystals exhibited tunable radioluminescence properties with high light yield under excitation from X-rays or gammarays, ${ }^{121,122}$ which enable them as high-performance X-ray scintillators. Heo et al. fabricated X-ray scintillators based on allinorganic perovskite $\mathrm{CsPbBr}_{3}$ nanoparticles, ${ }^{123}$ which comprised a carbon fiber reinforced polymer (CFRP), $\mathrm{CsPbBr}_{3}$ nanoparticles, and Si-PD arrays (as shown in Fig. 11a and b). The spatial resolution of the X-ray detector is $9.8 \mathrm{lp} \mathrm{mm}^{-1}$, which is almost the same as that of the commercial $\mathrm{CsI}(\mathrm{Tl})$ scintillator (10 $\mathrm{lp} \mathrm{mm}^{-1}$ ) and is much higher than that of gadolinium oxysulfide (GOS) scintillator $\left(6.2 \mathrm{lp} \mathrm{mm}^{-1}\right)$. Fig. 11c shows the $\mathrm{X}$-ray detection images obtained from both the $\mathrm{CsPbBr}_{3}$-based scintillator and the GOS-based scintillator. Even with decreasing $\mathrm{X}$-ray dose, the $\mathrm{CsPbBr}_{3}$-based scintillators can still hold the good image contrast as compared to the GOS-based scintillators. Recently, flexible X-ray detectors based on $\mathrm{CsPbBr}_{3}$ QDs were also fabricated on photomultiplier tube (PMT) substrates via a facile spin-coating method. ${ }^{124}$ As shown in Fig. 11d, the X-ray detectors exhibited a clear linear radioluminescence in relation with different X-ray dose rates. Moreover, a lowest detectable dose rate of 13 nGyair $^{-1}$ from these detection devices was achieved, which is over 400-times lower than the medical diagnostic baseline. Fig. 11e exhibits the real-time X-ray imaging setup of a flexible detector for a biological sample, where steel can be clearly found inside the insect body (as shown in Fig. 11f). Except for the all-inorganic perovskite QDs, $\mathrm{CsPbr}_{3}$ nanosheets with a high X-ray excited light-yield could also be fabricated into $\mathrm{X}$-ray scintillators. Zhang et al. reported a large-scale $\left(72 \mathrm{~cm}^{2}\right)$ $\mathrm{X}$-ray scintillator based on $\mathrm{CsPbBr}_{3}$ nanosheets, ${ }^{125}$ which showed a good image resolution of $0.21 \mathrm{~mm}$. Lead-based halide perovskites have already exhibited their outstanding capacity for both $\mathrm{X}$-ray detection and imaging in the past few years, keeping the gap between them and the commercial detectors more and more narrowing. Table 4 shows the key parameter comparison of currently reported halide perovskite-based X-ray detectors. 
a

C

e
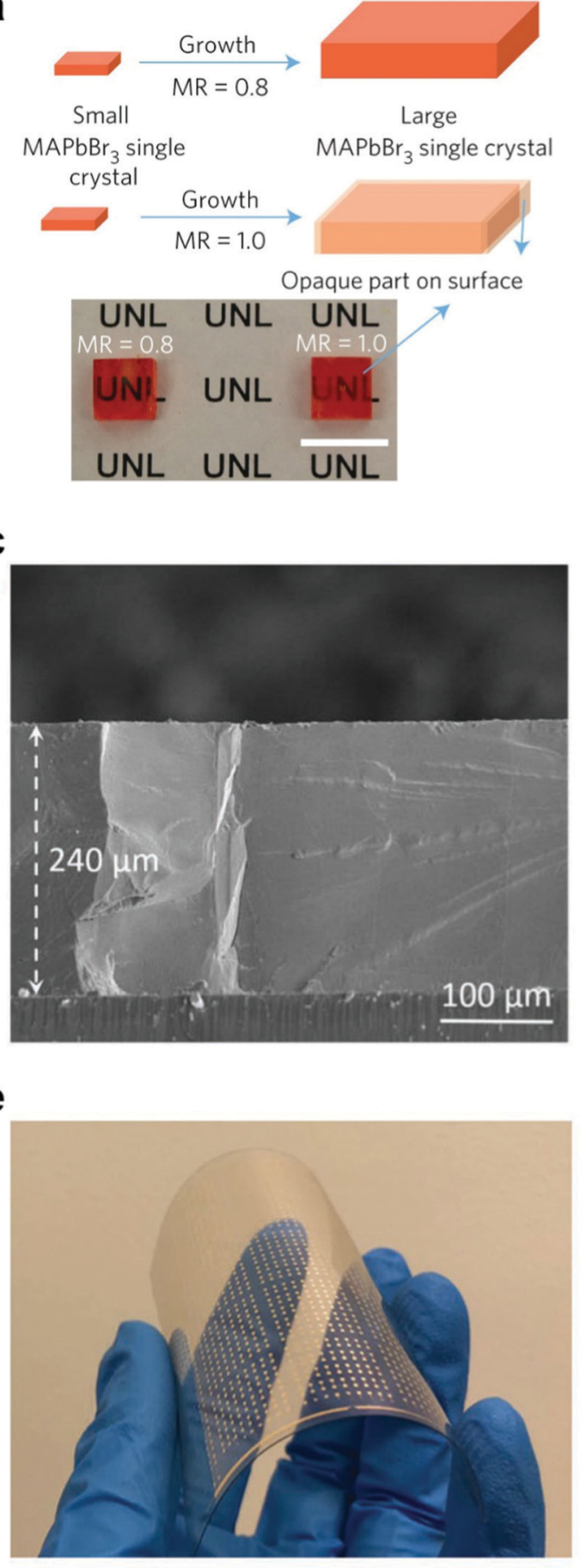

b
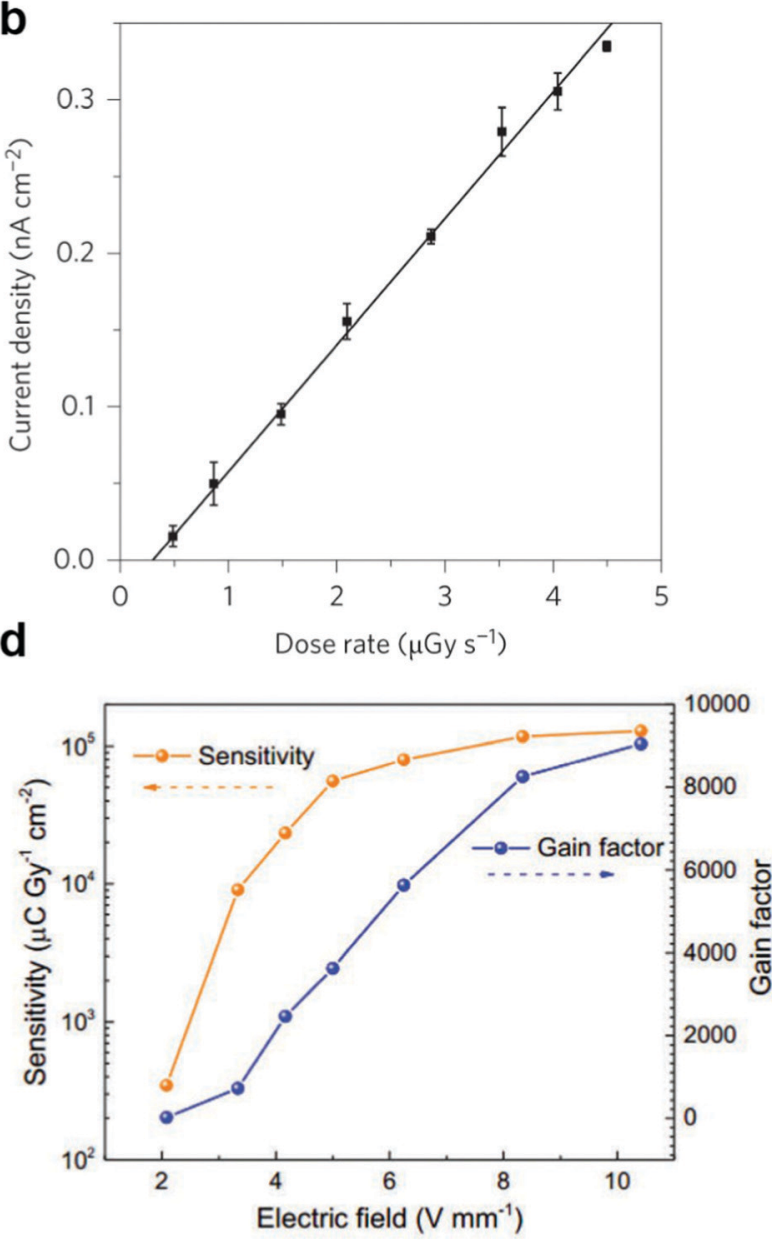

f

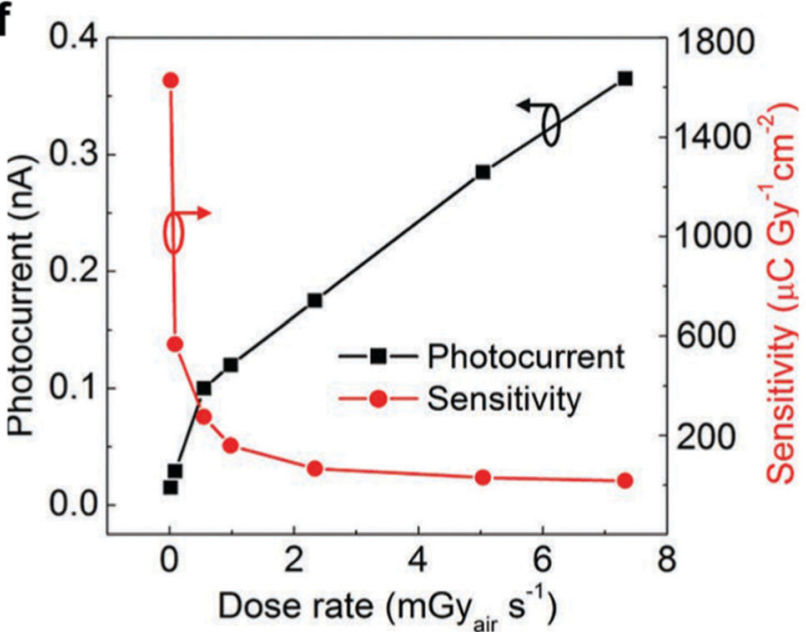

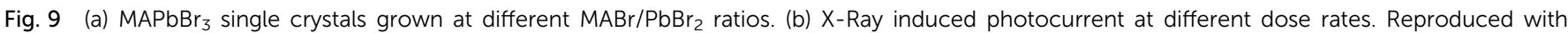

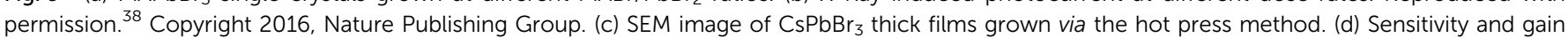

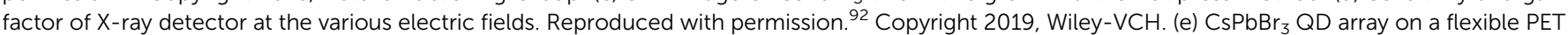
substrate. (f) X-Ray excited photocurrent and sensitivity at different dose rates. Reproduced with permission. ${ }^{119} \mathrm{Copyright} 2019$, Wiley-VCH.

\section{Non-lead halide perovskites for radiation detection}

Except for the lead halide perovskite-based radiation detectors, non-lead halide perovskites have also attracted enormous research interests due to their environmentally safe nature. For example, the double perovskites $\left(\mathrm{A}_{2} \mathrm{BB}^{\prime} \mathrm{X}_{6}\right)$ and the defect perovskites $\left(\mathrm{A}_{3} \mathrm{M}_{2} \mathrm{X}_{9}\right)$ have also shown a huge potential for radiation detection applications, in which the common $\mathrm{Pb}$ component is usually replaced by other heavy elements, such 
a

b

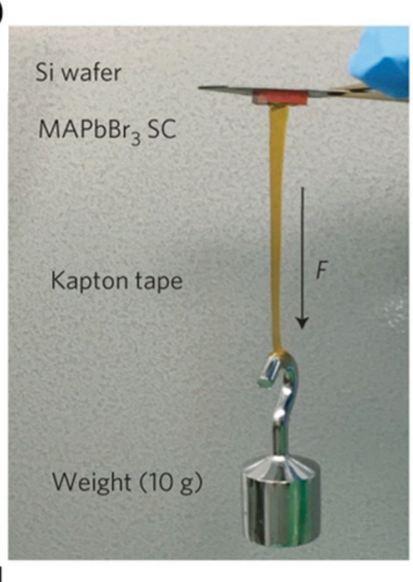

d

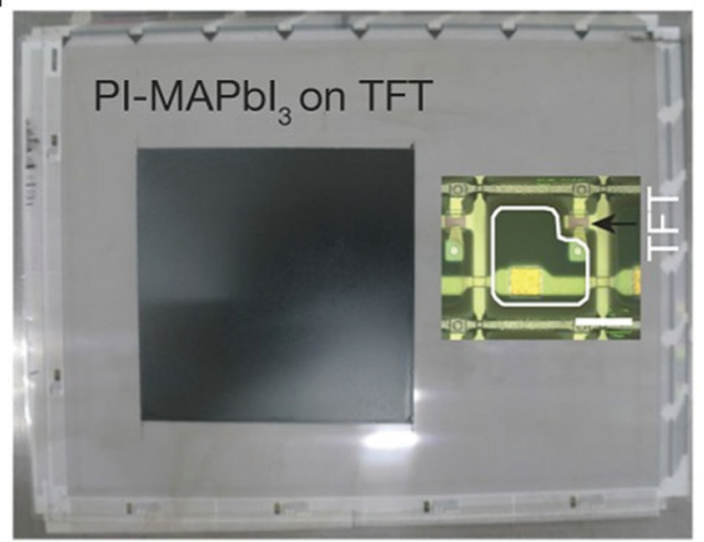

C
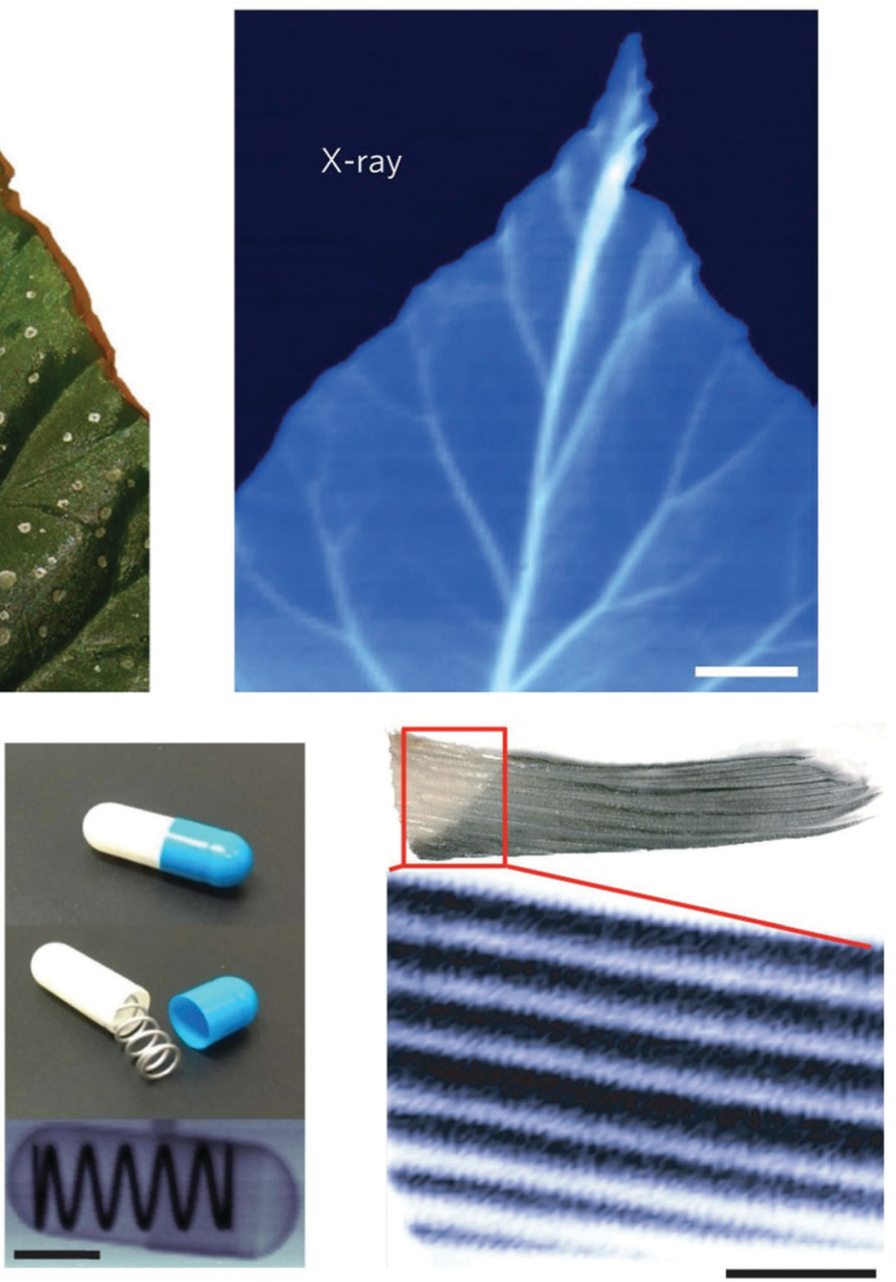

e

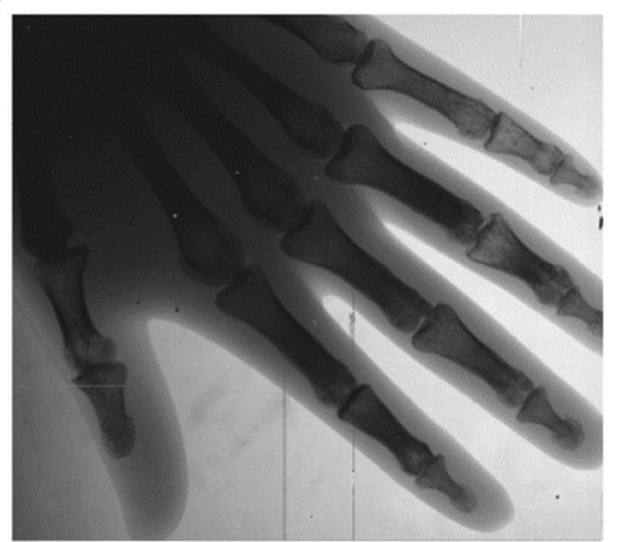

Fig. 10 (a) Photograph (left) and the corresponding X-ray image (right) obtained using a MAPbl 3 photodetector. Reproduced with permission. ${ }^{88}$ Copyright 2016, Nature Publishing Group. (b) Solid connection between $\mathrm{MAPbBr}_{3}$ single crystal and Si wafter integrated by a small molecule. (c) Optical and X-ray images of a capsule with a steel spring and a fish fin. Reproduced with permission. ${ }^{120}$ Copyright 2017, Nature Publishing Group. (d) Optical image of $\mathrm{MAPbl}_{3}$ on TFT backplane. Inset shows a pixel structure of TFT. (e) A hand phantom X-ray image was obtained using a MAPbl 3 photodetector. Reproduced with permission. ${ }^{36}$ Copyright 2017, Nature Publishing Group.

as $\mathrm{Bi}, \mathrm{Sb}$, and Ag. ${ }^{126-129}$ McCall et al. synthesized $\mathrm{A}_{3} \mathrm{M}_{2} \mathrm{I}_{9}(\mathrm{~A}=\mathrm{Cs}$, $\mathrm{Rb} ; \mathrm{B}=\mathrm{Bi}, \mathrm{Sb})$ defect perovskites for alpha-particle detection. ${ }^{130}$ These perovskites all have high average atomic numbers from 49 to 58 , which offer them enough attenuation coefficients and high stopping powers for radiation detection. However, it should be noted that these perovskite materials also have their own disadvantages, that is, the $\mu \tau$ products of these perovskites are only around $10^{-5} \mathrm{~cm}^{2} \mathrm{~V}^{-1}$. In this regard, replacing the inorganic elements $\mathrm{Cs}$ and $\mathrm{Rb}$ with $\mathrm{NH}_{4}$ group would be an effective approach. Zhuang et al. fabricated highly sensitive 
a

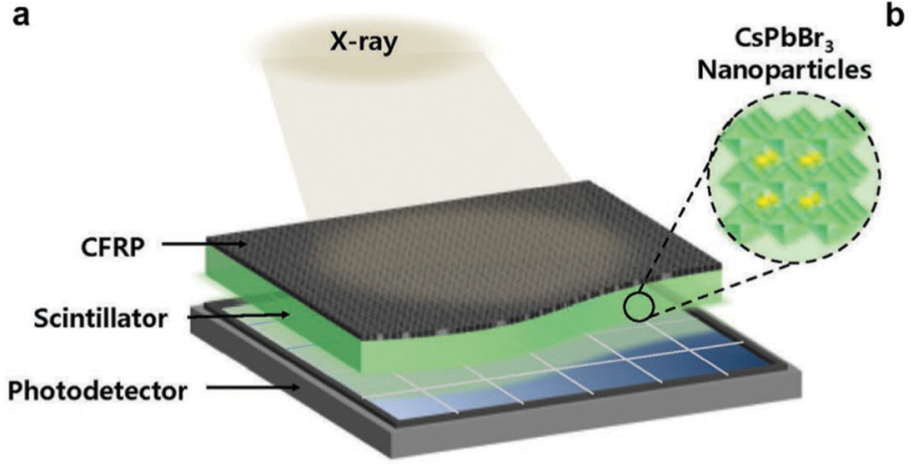

b

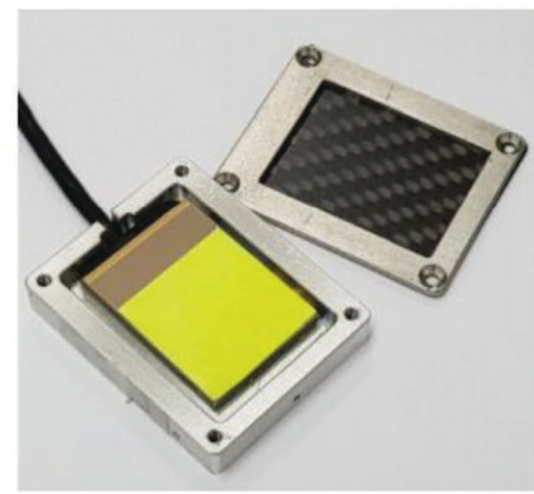

C
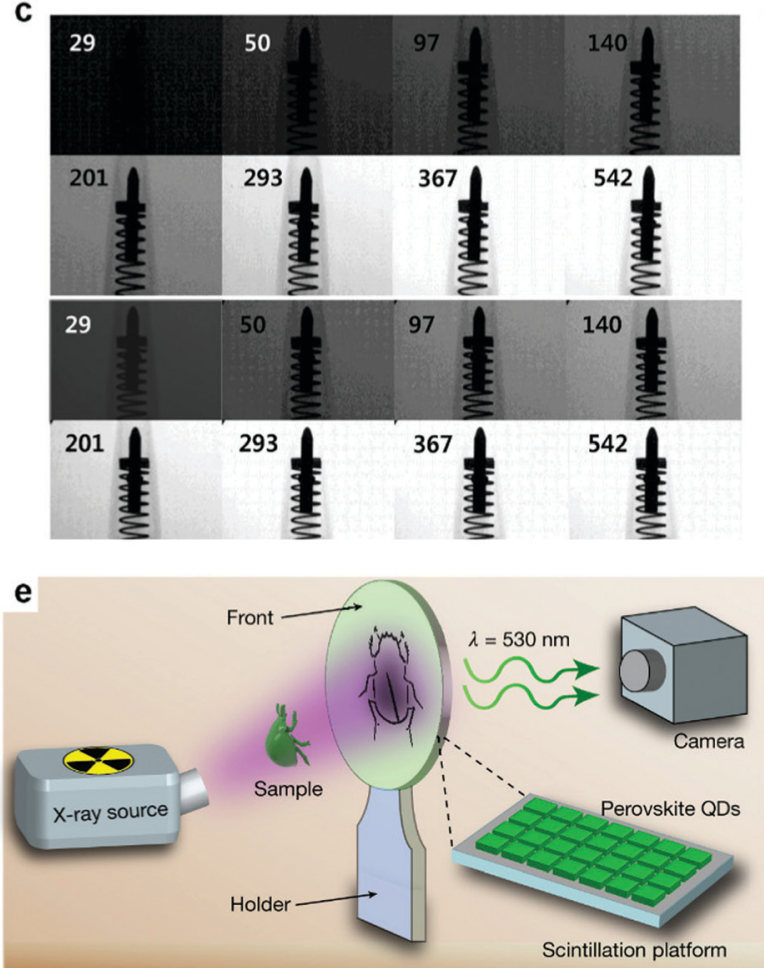

d
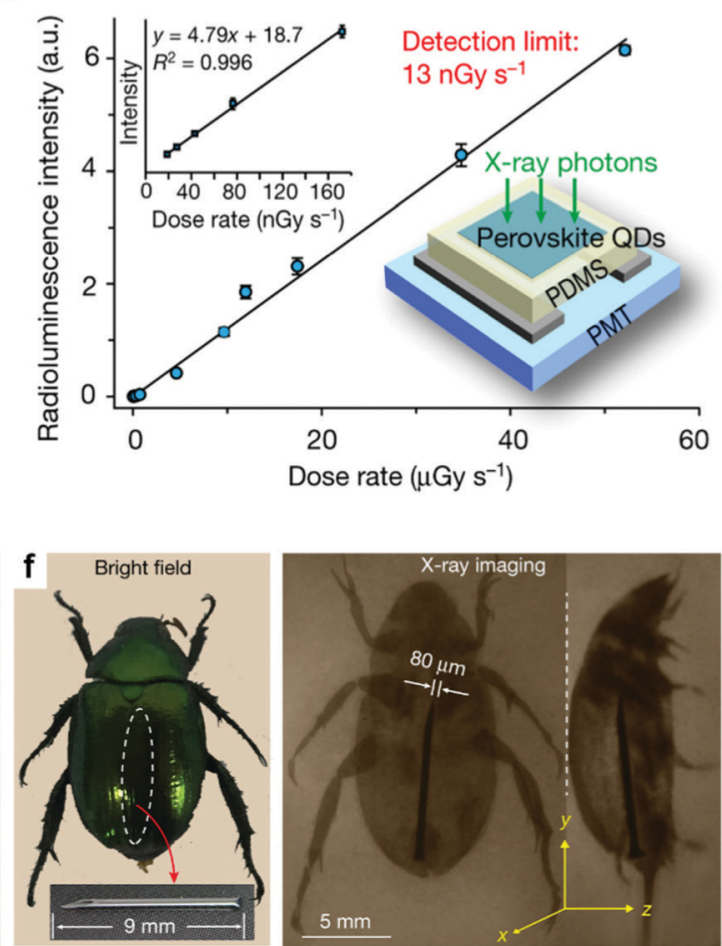

Fig. 11 (a) Schematic illustration of the CFRP/CsPbBr $3 / \mathrm{SiPD}$ X-ray detector. (b) Photograph of the $\mathrm{CsPbBr}$ based X-ray detector. (c) X-Ray images obtained using the GOS scintillator (top) and $\mathrm{CsPbBr}_{3}$ detector (bottom) under different X-ray dose rates. Reproduced with permission. ${ }^{123} \mathrm{Copyright} 2018$, Wiley-VCH. (d) Radioluminescence intensity of a flexible X-ray scintillator at different dose rates. (e) Set up for real-time X-ray diagnosis of biological samples. (f) Bright-field image (left) and the corresponding X-ray image (right) of the biosample. Reproduced with permission. ${ }^{124}$ Copyright 2018, Nature Publishing Group.

X-ray detectors based on $\left(\mathrm{NH}_{4}\right)_{3} \mathrm{Bi}_{2} \mathrm{I}_{9}$ single crystals. ${ }^{131}$ Encouragingly, the $\left(\mathrm{NH}_{4}\right)_{3} \mathrm{Bi}_{2} \mathrm{I}_{9}$ single crystals held a high $\mu \tau$ product of $10^{-2} \mathrm{~cm}^{2} \mathrm{~V}^{-1}$, which is comparable to those of $\mathrm{MAPbBr}_{3}$ and CZT single crystals. The relevant X-ray photodetector showed a lowest detectable dose rate of $55 \mathrm{nGy}_{\text {air }} \mathrm{s}^{-1}$. Zheng et al. have reported an X-ray detector based on $\mathrm{MA}_{3} \mathrm{Bi}_{2} \mathrm{I}_{9}$ single crystal with a much lower detectable limit of $0.62 \mathrm{nGy}_{\text {air }} \mathrm{s}^{-1}{ }^{132}$ (Fig. 12a and b). The anisotropic properties of $\mathrm{MA}_{3} \mathrm{Bi}_{2} \mathrm{I}_{9}$ single crystals led to a large resistance in the out-of-plane direction, by which a high sensitivity of $10620 \mu \mathrm{C} \mathrm{Gy}_{a i r}{ }^{-1} \mathrm{~cm}^{-2}$ was achieved. Double perovskite $\mathrm{Cs}_{2} \mathrm{AgBiBr}_{6}$ has also attracted huge research attention for X-ray detection, due to its high average atomic number of 53.1. Pan et al. reported $\mathrm{Cs}_{2} \mathrm{AgBiBr}_{6}$ single crystals with the large bulk resistances in the range of $10^{9}$ $10^{10} \Omega \mathrm{cm},{ }^{133}$ which further suppressed the dark current when applied in X-ray detectors. The all-inorganic components also enhanced thermostability, by which the single crystals can endure thermal stress up to $430{ }^{\circ} \mathrm{C}$ (in Fig. 12c). As shown in Fig. 12d, the lowest detectable limit of the related X-ray detectors is $59.7 \mathrm{nGy}_{\text {air }} \mathrm{s}^{-1}$ with a $\mu \tau$ product of $10^{-3} \mathrm{~cm}^{2} \mathrm{~V}^{-1}$. But, it should be pointed out that the sensitivity of $\mathrm{Cs}_{2} \mathrm{AgBiBr}_{6}$-based X-ray detector is relatively low $\left(105 \mu \mathrm{C} \mathrm{Gy}_{\mathrm{air}}{ }^{-1} \mathrm{~cm}^{-2}\right)$. More recently, the photophysical behavior of the $\mathrm{Cs}_{2} \mathrm{AgBiBr}_{6} \mathrm{X}$-ray detector has been studied by Steele et al., ${ }^{134}$ where they found that the radiationinduced charge carriers were less susceptible to scattering at lower temperatures and the resistance of $\mathrm{Cs}_{2} \mathrm{AgBiBr}_{6}$ was significantly increased as well, thus an improved device sensitivity of $2100 \mu \mathrm{C} \mathrm{Gy}_{\text {air }}{ }^{-1} \mathrm{~cm}^{-2}$ was obtained. These nonlead perovskite-based radiation detectors have shown their promising potential for radiation detection application, which 
Table 4 Figures of merit of a perovskite-based X-ray detector

\begin{tabular}{|c|c|c|c|c|c|}
\hline Material & $\mu \tau$ product $\left(\mathrm{cm}^{2} \mathrm{~V}^{-1}\right)$ & $\begin{array}{l}\text { Sensitivity } \\
\left(\mu \mathrm{C} \mathrm{Gy}_{\text {air }}{ }^{-1} \mathrm{~cm}^{-2}\right)\end{array}$ & $\begin{array}{l}\text { Lowest detectable } \\
\text { limit }\left(\mathrm{Gy}_{\mathrm{air}} \mathrm{s}^{-1}\right)\end{array}$ & $\begin{array}{l}\text { Spatial resolution } \\
\left(\mathrm{lp} \mathrm{mm}^{-1}\right)\end{array}$ & Ref. \\
\hline $\mathrm{MAPbBr}_{3}$ single crystal & $1.2 \times 10^{-2}$ & 80 & $0.5 \mu$ & - & 38 \\
\hline $\mathrm{MAPbI}_{3}$ polycrystal film & $2 \times 10^{-4}$ & 2527 & & - & 117 \\
\hline $\mathrm{CsPbBr}_{3}$ polycrystal film & - & 470 & $0.05 \mu$ & - & 118 \\
\hline $\mathrm{CsPbBr}_{3} \mathrm{QD}$ arrays & - & 1450 & $17.2 \mu$ & - & 119 \\
\hline $\mathrm{MAPbBr}_{3}$ single crystal & $4 \times 10^{-3}$ & 21000 & $36 \mathrm{n}$ & 10 & 120 \\
\hline
\end{tabular}
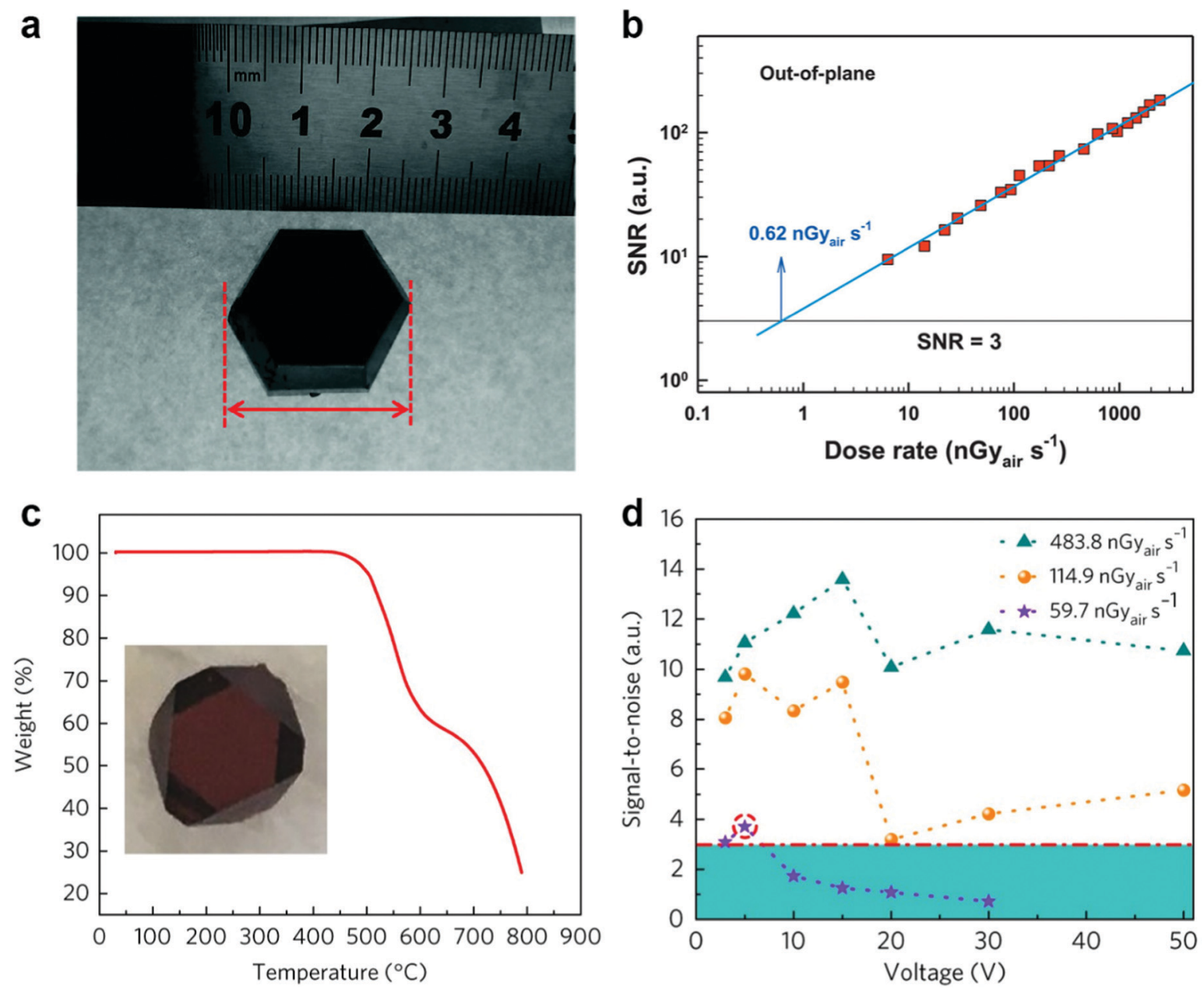

Fig. 12 (a) Photograph of $\mathrm{MA}_{3} \mathrm{Bi}_{2} \mathrm{l}_{9}$ single crystals. (b) X-Ray dose-dependent signal-to-noise ratio of the out-of-plane device with the lowest detectable dose of $0.62 \mathrm{nGy}_{\text {air }} \mathrm{S}^{-1}$. Reproduced with permission. ${ }^{132}$ Copyright 2020, Elsevier. (c) Thermogravimetric analysis of $\mathrm{Cs}_{2} \mathrm{AgBiBr}_{6}$. Included is a photograph of the $\mathrm{Cs}_{2} \mathrm{AgBiBr}_{6}$ single crystal. (d) The signal-to-noise ratio of the device under different voltages and X-ray doses. Reproduced with permission. ${ }^{133}$ Copyright 2017, Nature Publishing Group.

may further make the environmentally-safe halide perovskite detectors highly possible.

\section{Challenges and outlook}

Metal-halide perovskites have attracted tremendous research attention for various energy-related and optoelectronic applications in the past decade, due to their high absorption coefficients, long diffusion lengths, superb light yields, and high carrier mobilities. So far, many halide perovskite-based radiation detectors have also been reported, because of their fast and excellent response to the charged particles and photon radiation. These outstanding photophysical and transport properties of metal halide perovskites enable them as direct radiation detectors with high sensitivity and spectral resolution. Besides, metal halide perovskites can also be fabricated into scintillators working in the indirect mode due to their efficient light-emitting features under all types of 
radiation. Notably, metal halide perovskites can be easily integrated with other semiconductors for radiation detectors, which even hold superior device performance when appropriate device design is applied. Moreover, compared with today's commercial radiation detectors, like X-ray imagers, the detectors based on halide perovskites are far more sensitive and use less power. The low-cost raw materials and facile film and the crystal growth methods, mainly solution-processed approaches, will potentially reduce the fabrication and operation costs of X-ray detectors. Therefore, halide perovskite radiation detectors will offer better prices than the commercial radiation detectors that we use now.

Despite the advantages that metal halide perovskites hold for radiation detection applications, there are still a few challenges that block the commercialization of this class of materials. Firstly, the large-scale production of halide perovskites is still a daunting challenge and thus limits industrial-level radiation detector fabrication. Most commercial digital radiography detectors require an area larger than $1000 \mathrm{~cm}^{2}$, while the area of a large-scale perovskite film has just passed over A4 size. ${ }^{135}$ Even though the scalable fabrication of perovskite film is technically achievable, the accompanying trade-off on device performance is still unaffordable. Then, there is still a vast distance in the detection performance between halide perovskite detectors and the existing commercial radiation detectors. As for alpha particle detection, the state-of-the-art detector offers the highest spectral resolution of $0.25 \%,{ }^{136}$ while attempts to detect alpha particles using halide perovskites are still in the early stages with a spectral resolution of only $15 \%$. The main reason is that the resistance of halide perovskites $\left(10^{7}-10^{9} \Omega \mathrm{cm}\right)$ is still not high enough to reduce the dark current compared with the high resistance of $10^{11} \Omega \mathrm{cm}$ for the $4 \mathrm{H}-\mathrm{SiC}$ epitaxial layer, which may reduce the signal quality at high voltage bias and thus lower the spectral resolution. On the one hand, building an asymmetric device structure induces a contact barrier which can sufficiently reduce the device's dark current. On the other hand, how to reduce the grain boundaries and intrinsic defects on perovskite films and single crystals is also another concern on suppressing the relatively high dark currents. Furthermore, as an electronic-ionic mixed material, severe ion migration occurs in perovskite materials under applied voltage which can even react with the electrodes and lead to the decomposition of the perovskite active layer. ${ }^{137-139}$ Effective reduction of ion migration in halide perovskites is essential for sustaining detector performance, which may require a deeper understanding of the physical mechanism inside halide perovskites. Plus, the stability of halide perovskites needs to be taken into consideration because most of them suffer from decomposition in the air, especially the hybrid perovskites in which the organic parts are chemically active. ${ }^{140}$ Currently several methods have been developed for improving the long-term stability of halide perovskite, such as surface engineering, passivation of grain boundaries, construction of heterostructures, and so on, while the thermal stability and phase stability cannot be satisfied in both ways yet. Although the all-inorganic halide perovskites show better longterm stability in the air, a thorough encapsulation method is still in high demand. Environmental safety would be another concern for commercial radiation applications based on halide perovskite, particularly the lead-based perovskites, due to the presence of the heavy metal component $\mathrm{Pb}$. A totally environment-friendly assembly line is required for perovskite radiation detectors to minimize lead contamination.

Regarding the photon radiation detections, the highest gamma-ray spectral resolution is only $0.5 \%$ which was obtained in the commercial CZT crystal-based detectors at room temperature. Although impressive progress on metal halide perovskite-based gamma-ray detectors has been made, the spectral peak resolution is still far low from this level, which can be attributed to the relatively higher dark current, especially for the lead iodide types. One of the reasons is the self-doping nature of single halide component perovskites, which causes the perovskites to demonstrate $\mathrm{p}$ - or n-type and thus lowers their resistances. Though the self-doping of perovskite can be tuned by adjusting the halide component ratio, new phase purity and decreasing mobility will arise as new obstacles. Besides, the longterm stability of halide perovskites under the radiation of gamma-rays remains elusive, where the organic components in hybrid perovskites may suffer degradation. Metal halide perovskite-based direct X-ray detectors share the same challenges as those of gamma-ray detectors. However, due to the imaging requirement, the lowest detectable limit and spatial resolution should be taken into consideration as extra factors. On the one hand, the lowest detectable limit of halide perovskite-based X-ray detectors keeps renewing which is enough for medical diagnosis now. On the other hand, larger detectors are required for imaging under a lower X-ray dose rate while the resolution decreases as the detector area increases. Moreover, the spatial resolution of direct $\mathrm{X}$-ray detectors is still left behind the first-class HPGe detector, which is limited by both the perovskites quality and device fabrication method.

Despite being confronted with these challenges, a bright future for metal halide perovskite-based radiation detection applications can be clearly seen, considering their tremendous advantages, including the low-cost raw materials, facile production, excellent compatibility, and superb optoelectronic properties. In addition, given the fact that the active halide perovskite crystalline films or quantum dots can be grown by solution-processing at a relatively low temperature, it would be quite possible to produce flexible radiation detectors for commercial applications. In this sense, halide perovskite $\mathrm{X}$-ray imagers could conform to whatever is being scanned. Therefore, these flexible features would enable halide perovskite radiation detectors to detect some diseases like cancer earlier. With more investigations on this promising field, halide perovskite-based radiation detectors, which show the high device performance in detecting both the high-energy particle radiation (alpha and beta particles) and the photon radiation (gamma-ray and X-ray), hold great potential to lead the replacement of the existing radiation detection devices, like $\mathrm{X}$-ray detection technologies, which are omnipresent in our daily life and used in a multitude of medical diagnostic and therapeutic tools, for security checking and safety inspections 
of luggage, or in various scientific equipment. Furthermore, as compared with today's commercial radiation devices, halide perovskite detectors are far more sensitive and use less power. Also, the low-cost raw materials and crystal growth will potentially reduce the fabrication and operation costs of X-ray detectors. Therefore, it can be reasonably estimated that halide perovskites will not only revolutionize the radiation detection applications but also expand what is already a multibilliondollar industry.

\section{Conflicts of interest}

There are no conflicts to declare.

\section{Acknowledgements}

The authors would like to acknowledge financial support from the Discovery Early Career Researcher Award (DECRA) scheme (DE180100167) from the Australian Research Council (ARC). Tiebin Yang acknowledges the support from the Dean of Science's International Postgraduate Research Scholarship. We would also like to thank the Research and Prototype Foundry (RPF), a Core Research Facility based at the Sydney Nanoscience Hub at the University of Sydney and a member of the ANFF network for providing a lot of support for our research work.

\section{References}

1 G. F. Knoll, Radiation Detection and Measurement, John Wiley \& Sons, 2010.

2 D. Chapman, W. Thomlinson, R. E. Johnston, D. Washburn, E. Pisano, N. Gmür, Z. Zhong, R. Menk, F. Arfelli and D. Sayers, Phys. Med. Biol., 1997, 42, 2015-2025.

3 F. El Ghissassi, R. Baan, K. Straif, Y. Grosse, B. Secretan, V. Bouvard, L. Benbrahim-Tallaa, N. Guha, C. Freeman, L. Galichet and V. Cogliano, Lancet Oncol., 2009, 10, 751-752.

4 H. N. Chapman, P. Fromme, A. Barty, T. A. White, R. A. Kirian, A. Aquila, M. S. Hunter, J. Schulz, D. P. DePonte, U. Weierstall, R. B. Doak, F. R. N. C. Maia, A. V. Martin, I. Schlichting, L. Lomb, N. Coppola, R. L. Shoeman, S. W. Epp, R. Hartmann, D. Rolles, A. Rudenko, L. Foucar, N. Kimmel, G. Weidenspointner, P. Holl, M. Liang, M. Barthelmess, C. Caleman, S. Boutet, M. J. Bogan, J. Krzywinski, C. Bostedt, S. Bajt, L. Gumprecht, B. Rudek, B. Erk, C. Schmidt, A. Hömke, C. Reich, D. Pietschner, L. Strüder, G. Hauser, H. Gorke, J. Ullrich, S. Herrmann, G. Schaller, F. Schopper, H. Soltau, K.-U. Kühnel, M. Messerschmidt, J. D. Bozek, S. P. Hau-Riege, M. Frank, C. Y. Hampton, R. G. Sierra, D. Starodub, G. J. Williams, J. Hajdu, N. Timneanu, M. M. Seibert, J. Andreasson, A. Rocker, O. Jönsson, M. Svenda, S. Stern, K. Nass, R. Andritschke, C.-D. Schröter, F. Krasniqi, M. Bott, K. E. Schmidt, X. Wang, I. Grotjohann, J. M. Holton,
T. R. M. Barends, R. Neutze, S. Marchesini, R. Fromme, S. Schorb, D. Rupp, M. Adolph, T. Gorkhover, I. Andersson, H. Hirsemann, G. Potdevin, H. Graafsma, B. Nilsson and J. C. H. Spence, Nature, 2011, 470, 73-77.

5 R. P. Haff and N. Toyofuku, Sens. Instrum. Food Qual., 2008, 2, 262-273.

6 F. Arfelli, M. Assante, V. Bonvicini, A. Bravin, G. Cantatore, E. Castelli, L. D. Palma, M. D. Michiel, R. Longo, A. Olivo, S. Pani, D. Pontoni, P. Poropat, M. Prest, A. Rashevsky, G. Tromba, A. Vacchi, E. Vallazza and F. Zanconati, Phys. Med. Biol., 1998, 43, 2845-2852.

7 Y. Eisen, A. Shor and I. Mardor, Nucl. Instrum. Methods Phys. Res., Sect. A, 1999, 428, 158-170.

8 M. J. Yaffe and J. A. Rowlands, Phys. Med. Biol., 1997, 42, $1-39$.

9 A. Owens and A. Peacock, Nucl. Instrum. Methods Phys. Res., Sect. A, 2004, 531, 18-37.

10 S. Kasap, J. B. Frey, G. Belev, O. Tousignant, H. Mani, J. Greenspan, L. Laperriere, O. Bubon, A. Reznik, G. DeCrescenzo, K. S. Karim and J. A. Rowlands, Sensors, 2011, 11, 5112-5157.

11 M. Guerra, M. Manso, S. Longelin, S. Pessanha and M. L. Carvalho, J. Inst., 2012, 7, C10004.

12 S. O. Kasap, J. Phys. D: Appl. Phys., 2000, 33, 2853-2865.

13 P. J. Sellin, Nucl. Instrum. Methods Phys. Res., Sect. A, 2003, 513, 332-339.

14 C. Szeles, Phys. Status Solidi B, 2004, 241, 783-790.

15 T. E. Schlesinger, J. E. Toney, H. Yoon, E. Y. Lee, B. A. Brunett, L. Franks and R. B. James, Mater. Sci. Eng., $R$, 2001, 32, 103-189.

16 M. Moszyński, A. Nassalski, A. Syntfeld-Każuch, T. Szczęśniak, W. Czarnacki, D. Wolski, G. Pausch and J. Stein, Nucl. Instrum. Methods Phys. Res., Sect. A, 2006, 568, 739-751.

17 G. Bizarri, J. de Haas, P. Dorenbos and C. W. E. Eijk, IEEE Trans. Nucl. Sci., 2006, 53, 615-619.

18 V. B. Mikhailik, V. Kapustyanyk, V. Tsybulskyi, V. Rudyk and H. Kraus, Phys. Status Solidi B, 2015, 252, 804-810.

19 N. J. Jeon, J. H. Noh, Y. C. Kim, W. S. Yang, S. Ryu and S. I. Seok, Nat. Mater., 2014, 13, 897-903.

20 X. Li, D. Bi, C. Yi, J.-D. Décoppet, J. Luo, S. M. Zakeeruddin, A. Hagfeldt and M. Grätzel, Science, 2016, 353, 58-62.

21 A. Al-Ashouri, E. Köhnen, B. Li, A. Magomedov, H. Hempel, P. Caprioglio, J. A. Márquez, A. B. M. Vilches, E. Kasparavicius, J. A. Smith, N. Phung, D. Menzel, M. Grischek, L. Kegelmann, D. Skroblin, C. Gollwitzer, T. Malinauskas, M. Jošt, G. Matič, B. Rech, R. Schlatmann, M. Topič, L. Korte, A. Abate, B. Stannowski, D. Neher, M. Stolterfoht, T. Unold, V. Getautis and S. Albrecht, Science, 2020, 370, 1300-1309.

22 Z.-K. Tan, R. S. Moghaddam, M. L. Lai, P. Docampo, R. Higler, F. Deschler, M. Price, A. Sadhanala, L. M. Pazos, D. Credgington, F. Hanusch, T. Bein, H. J. Snaith and R. H. Friend, Nat. Nanotechnol., 2014, 9, 687-692.

23 H. Cho, S.-H. Jeong, M.-H. Park, Y.-H. Kim, C. Wolf, C.-L. Lee, J. H. Heo, A. Sadhanala, N. Myoung, S. Yoo, 
S. H. Im, R. H. Friend and T.-W. Lee, Science, 2015, 350, 1222-1225.

24 Z. Xiao, R. A. Kerner, L. Zhao, N. L. Tran, K. M. Lee, T.-W. Koh, G. D. Scholes and B. P. Rand, Nat. Photonics, 2017, 11, 108.

25 B. R. Sutherland and E. H. Sargent, Nat. Photonics, 2016, 10, 295-302.

26 F. Li, C. Ma, H. Wang, W. Hu, W. Yu, A. D. Sheikh and T. Wu, Nat. Commun., 2015, 6, 8238.

27 C. Huo, X. Liu, X. Song, Z. Wang and H. Zeng, J. Phys. Chem. Lett., 2017, 8, 4785-4792.

28 Y. Zou, F. Li, C. Zhao, J. Xing, Z. Yu, W. Yu and C. Guo, Adv. Opt. Mater., 2019, 7, 1900676.

29 X. Y. Chin, D. Cortecchia, J. Yin, A. Bruno and C. Soci, Nat. Commun., 2015, 6, 7383.

30 X. Liu, D. Yu, F. Cao, X. Li, J. Ji, J. Chen, X. Song and H. Zeng, Small, 2017, 13, 1700364.

31 F. P. G. de Arquer, A. Armin, P. Meredith and E. H. Sargent, Nat. Rev. Mater., 2017, 2, 16100.

32 J. Song, Q. Cui, J. Li, J. Xu, Y. Wang, L. Xu, J. Xue, Y. Dong, T. Tian, H. Sun and H. Zeng, Adv. Opt. Mater., 2017, 5, 1700157.

33 Z. Sun, L. Aigouy and Z. Chen, Nanoscale, 2016, 8, 7377-7383.

34 C. C. Stoumpos, C. D. Malliakas, J. A. Peters, Z. Liu, M. Sebastian, J. Im, T. C. Chasapis, A. C. Wibowo, D. Y. Chung, A. J. Freeman, B. W. Wessels and M. G. Kanatzidis, Cryst. Growth Des., 2013, 13, 2722-2727.

35 Y. He, L. Matei, H. J. Jung, K. M. McCall, M. Chen, C. C. Stoumpos, Z. Liu, J. A. Peters, D. Y. Chung, B. W. Wessels, M. R. Wasielewski, V. P. Dravid, A. Burger and M. G. Kanatzidis, Nat. Commun., 2018, 9, 1609.

36 Y. C. Kim, K. H. Kim, D.-Y. Son, D.-N. Jeong, J.-Y. Seo, Y. S. Choi, I. T. Han, S. Y. Lee and N.-G. Park, Nature, 2017, 550, 87-91.

37 D. Yu, P. Wang, F. Cao, Y. Gu, J. Liu, Z. Han, B. Huang, Y. Zou, X. Xu and H. Zeng, Nat. Commun., 2020, 11, 3395.

38 H. Wei, Y. Fang, P. Mulligan, W. Chuirazzi, H.-H. Fang, C. Wang, B. R. Ecker, Y. Gao, M. A. Loi, L. Cao and J. Huang, Nat. Photonics, 2016, 10, 333-339.

39 H. Wei and J. Huang, Nat. Commun., 2019, 10, 1066.

40 L. Gao and Q. Yan, Sol. RRL, 2020, 4, 1900210.

41 G. Kakavelakis, M. Gedda, A. Panagiotopoulos, E. Kymakis,

T. D. Anthopoulos and K. Petridis, Adv. Sci., 2020, 7, 2002098.

42 S. Del Sordo, L. Abbene, E. Caroli, A. M. Mancini, A. Zappettini and P. Ubertini, Sensors, 2009, 9, 3491-3526.

43 J. D. Eskin, H. H. Barrett and H. B. Barber, J. Appl. Phys., 1998, 85, 647-659.

44 M. Richter and P. Siffert, Nucl. Instrum. Methods Phys. Res., Sect. A, 1992, 322, 529-537.

45 J. Androulakis, S. C. Peter, H. Li, C. D. Malliakas, J. A. Peters, Z. Liu, B. W. Wessels, J.-H. Song, H. Jin, A. J. Freeman and M. G. Kanatzidis, Adv. Mater., 2011, 23, 4163-4167.

46 V. F. Dvoryankin, G. G. Dvoryankina, A. A. Kudryashov, A. G. Petrov, V. D. Golyshev and S. V. Bykova, Tech. Phys., 2010, 55, 306-308.
47 P. Büchele, M. Richter, S. F. Tedde, G. J. Matt, G. N. Ankah, R. Fischer, M. Biele, W. Metzger, S. Lilliu, O. Bikondoa, J. E. Macdonald, C. J. Brabec, T. Kraus, U. Lemmer and O. Schmidt, Nat. Photonics, 2015, 9, 843-848.

48 L. Protesescu, S. Yakunin, M. I. Bodnarchuk, F. Krieg, R. Caputo, C. H. Hendon, R. X. Yang, A. Walsh and M. V. Kovalenko, Nano Lett., 2015, 15, 3692-3696.

49 L. Dou, Y. (Micheal) Yang, J. You, Z. Hong, W.-H. Chang, G. Li and Y. Yang, Nat. Commun., 2014, 5, 5404.

50 M. V. Kovalenko, L. Protesescu and M. I. Bodnarchuk, Science, 2017, 358, 745-750.

51 M. I. Saidaminov, M. A. Haque, J. Almutlaq, S. Sarmah, X.-H. Miao, R. Begum, A. A. Zhumekenov, I. Dursun, N. Cho, B. Murali, O. F. Mohammed, T. Wu and O. M. Bakr, Adv. Opt. Mater., 2017, 5, 1600704.

52 M. I. Saidaminov, A. L. Abdelhady, B. Murali, E. Alarousu, V. M. Burlakov, W. Peng, I. Dursun, L. Wang, Y. He, G. Maculan, A. Goriely, T. Wu, O. F. Mohammed and O. M. Bakr, Nat. Commun., 2015, 6, 1-6.

53 Y. Cao, N. Wang, H. Tian, J. Guo, Y. Wei, H. Chen, Y. Miao, W. Zou, K. Pan, Y. He, H. Cao, Y. Ke, M. Xu, Y. Wang, M. Yang, K. Du, Z. Fu, D. Kong, D. Dai, Y. Jin, G. Li, H. Li, Q. Peng, J. Wang and W. Huang, Nature, 2018, 562, 249-253.

54 K. Lin, J. Xing, L. N. Quan, F. P. G. de Arquer, X. Gong, J. Lu, L. Xie, W. Zhao, D. Zhang, C. Yan, W. Li, X. Liu, Y. Lu, J. Kirman, E. H. Sargent, Q. Xiong and Z. Wei, Nature, 2018, 562, 245-248.

55 T. Chiba, Y. Hayashi, H. Ebe, K. Hoshi, J. Sato, S. Sato, Y.-J. Pu, S. Ohisa and J. Kido, Nat. Photonics, 2018, 12, 681-687.

56 X. Zhang, B. Xu, J. Zhang, Y. Gao, Y. Zheng, K. Wang and X. W. Sun, Adv. Funct. Mater., 2016, 26, 4595-4600.

57 H. Zhu, Y. Fu, F. Meng, X. Wu, Z. Gong, Q. Ding, M. V. Gustafsson, M. T. Trinh, S. Jin and X.-Y. Zhu, Nat. Mater., 2015, 14, 636-642.

58 A. Kojima, K. Teshima, Y. Shirai and T. Miyasaka, J. Am. Chem. Soc., 2009, 131, 6050-6051.

59 M. A. Green, A. Ho-Baillie and H. J. Snaith, Nat. Photonics, 2014, 8, 506-514.

60 W. Chen, Y. Wu, Y. Yue, J. Liu, W. Zhang, X. Yang, H. Chen, E. Bi, I. Ashraful, M. Grätzel and L. Han, Science, 2015, 350, 944-948.

61 H. Zhou, Q. Chen, G. Li, S. Luo, T. Song, H.-S. Duan, Z. Hong, J. You, Y. Liu and Y. Yang, Science, 2014, 345, 542-546.

62 Q. A. Akkerman, G. Rainò, M. V. Kovalenko and L. Manna, Nat. Mater., 2018, 17, 394.

63 X. Li, F. Cao, D. Yu, J. Chen, Z. Sun, Y. Shen, Y. Zhu, L. Wang, Y. Wei, Y. Wu and H. Zeng, Small, 2017, 13, 1603996.

64 T. Yang, F. Li and R. Zheng, ACS Appl. Electron. Mater., 2019, 1, 1348-1366.

65 Y. Fu, H. Zhu, J. Chen, M. P. Hautzinger, X.-Y. Zhu and S. Jin, Nat. Rev. Mater., 2019, 4, 169.

66 Y. Dong, Y. Zou, J. Song, X. Song and H. Zeng, J. Mater. Chem. C, 2017, 5, 11369-11394. 
67 X. Zhang, F. Li and R. Zheng, J. Mater. Chem. C, 2020, 8, 13918-13952.

68 W. Pan, H. Wei and B. Yang, Front. Chem., 2020, 8, 268.

69 R. Maruyama, Reference Module in Earth Systems and Environmental Sciences, Elsevier, 2021.

70 J.-W. Xiao, L. Liu, D. Zhang, N. D. Marco, J.-W. Lee, O. Lin, Q. Chen and Y. Yang, Adv. Energy Mater., 2017, 7, 1700491.

71 Q. A. Akkerman, S. G. Motti, A. R. Srimath Kandada, E. Mosconi, V. D’Innocenzo, G. Bertoni, S. Marras, B. A. Kamino, L. Miranda, F. De Angelis, A. Petrozza, M. Prato and L. Manna, J. Am. Chem. Soc., 2016, 138, 1010-1016.

72 H. Gao, J. Feng, Y. Pi, Z. Zhou, B. Zhang, Y. Wu, X. Wang, X. Jiang and L. Jiang, Adv. Funct. Mater., 2018, 28, 1804349.

73 D. Dong, H. Deng, C. Hu, H. Song, K. Qiao, X. Yang, J. Zhang, F. Cai, J. Tang and H. Song, Nanoscale, 2017, 9, 1567-1574.

74 D. Zhang, Y. Yang, Y. Bekenstein, Y. Yu, N. A. Gibson, A. B. Wong, S. W. Eaton, N. Kornienko, Q. Kong, M. Lai, A. P. Alivisatos, S. R. Leone and P. Yang, J. Am. Chem. Soc., 2016, 138, 7236-7239.

75 X.-J. She, C. Chen, G. Divitini, B. Zhao, Y. Li, J. Wang, J. F. Orri, L. Cui, W. Xu, J. Peng, S. Wang, A. Sadhanala and H. Sirringhaus, Nat. Electron., 2020, 3, 694-703.

76 Z. Lian, Q. Yan, T. Gao, J. Ding, Q. Lv, C. Ning, Q. Li and J. Sun, J. Am. Chem. Soc., 2016, 138, 9409-9412.

77 D. Shi, V. Adinolfi, R. Comin, M. Yuan, E. Alarousu, A. Buin, Y. Chen, S. Hoogland, A. Rothenberger, K. Katsiev, Y. Losovyj, X. Zhang, P. A. Dowben, O. F. Mohammed, E. H. Sargent and O. M. Bakr, Science, 2015, 347, 519-522.

78 G. Maculan, A. D. Sheikh, A. L. Abdelhady, M. I. Saidaminov, M. A. Haque, B. Murali, E. Alarousu, O. F. Mohammed, T. Wu and O. M. Bakr, J. Phys. Chem. Lett., 2015, 6, 3781-3786.

79 Q. Dong, Y. Fang, Y. Shao, P. Mulligan, J. Qiu, L. Cao and J. Huang, Science, 2015, 347, 967-970.

80 M. I. Saidaminov, V. Adinolfi, R. Comin, A. L. Abdelhady, W. Peng, I. Dursun, M. Yuan, S. Hoogland, E. H. Sargent and O. M. Bakr, Nat. Commun., 2015, 6, 8724.

81 Y. Rakita, N. Kedem, S. Gupta, A. Sadhanala, V. Kalchenko, M. L. Böhm, M. Kulbak, R. H. Friend, D. Cahen and G. Hodes, Cryst. Growth Des., 2016, 16, 5717-5725.

82 J. Song, J. Li, X. Li, L. Xu, Y. Dong and H. Zeng, Adv. Mater., 2015, 27, 7162-7167.

83 D. Yang, X. Li, W. Zhou, S. Zhang, C. Meng, Y. Wu, Y. Wang and H. Zeng, Adv. Mater., 2019, 31, 1900767.

84 V. B. Mykhaylyk, H. Kraus and M. Saliba, Mater. Horiz., 2019, 6, 1740-1747.

85 Q. A. Akkerman, V. D'Innocenzo, S. Accornero, A. Scarpellini, A. Petrozza, M. Prato and L. Manna, J. Am. Chem. Soc., 2015, 137, 10276-10281.

86 L. C. Schmidt, A. Pertegás, S. González-Carrero, O. Malinkiewicz, S. Agouram, G. Mínguez Espallargas, H. J. Bolink, R. E. Galian and J. Pérez-Prieto, J. Am. Chem. Soc., 2014, 136, 850-853.
87 M. V. Kovalenko, L. Protesescu and M. I. Bodnarchuk, Science, 2017, 358, 745-750.

88 S. Yakunin, M. Sytnyk, D. Kriegner, S. Shrestha, M. Richter, G. J. Matt, H. Azimi, C. J. Brabec, J. Stangl, M. V. Kovalenko and W. Heiss, Nat. Photonics, 2015, 9, 444-449.

89 H. Wei, D. DeSantis, W. Wei, Y. Deng, D. Guo, T. J. Savenije, L. Cao and J. Huang, Nat. Mater., 2017, 16, 826-833.

90 Q. Xu, J. Wang, W. Shao, X. Ouyang, X. Wang, X. Zhang, Y. Guo and X. Ouyang, Nanoscale, 2020, 12, 9727-9732.

91 Y. He, Z. Liu, K. M. McCall, W. Lin, D. Y. Chung, B. W. Wessels and M. G. Kanatzidis, Nucl. Instrum. Methods Phys. Res., Sect. A, 2019, 922, 217-221.

92 W. Pan, B. Yang, G. Niu, K.-H. Xue, X. Du, L. Yin, M. Zhang, H. Wu, X.-S. Miao and J. Tang, Adv. Mater., 2019, 31, 1904405.

93 W. Wang, H. Meng, H. Qi, H. Xu, W. Du, Y. Yang, Y. Yi, S. Jing, S. Xu, F. Hong, J. Qin, J. Huang, Z. Xu, Y. Zhu, R. Xu, J. Lai, F. Xu, L. Wang and J. Zhu, Adv. Mater., 2020, 32, 2001540.

94 W. Zhu, W. Ma, Y. Su, Z. Chen, X. Chen, Y. Ma, L. Bai, W. Xiao, T. Liu, H. Zhu, X. Liu, H. Liu, X. Liu and Y. (Michael) Yang, Light: Sci. Appl., 2020, 9, 112.

95 F. Zhou, Z. Li, W. Lan, Q. Wang, L. Ding and Z. Jin, Small Methods, 2020, 4, 2000506.

96 Y. Li, W. Shao, X. Ouyang, Z. Zhu, H. Zhang, X. Ouyang, B. Liu and Q. Xu, J. Phys. Chem. C, 2019, 123, 17449-17453.

97 E. Rutherford and T. Royds, Nature, 1908, 78, 220-221.

98 J. Grellier, W. Atkinson, P. Bérard, D. Bingham, A. Birchall, E. Blanchardon, R. Bull, I. Guseva Canu, C. Challeton-de Vathaire, R. Cockerill, M. T. Do, H. Engels, J. Figuerola, A. Foster, L. Holmstock, C. Hurtgen, D. Laurier, M. Puncher, A. E. Riddell, E. Samson, I. Thierry-Chef, M. Tirmarche, M. Vrijheid and E. Cardis, Epidemiology, 2017, 28, 675-684.

99 K. S. Shah, J. C. Lund, F. Olschner, L. Moy and M. R. Squillante, IEEE Trans. Nucl. Sci., 1989, 36, 199-202.

100 Y. He, O. Y. Kontsevoi, C. C. Stoumpos, G. G. Trimarchi, S. M. Islam, Z. Liu, S. S. Kostina, S. Das, J.-I. Kim, W. Lin, B. W. Wessels and M. G. Kanatzidis, J. Am. Chem. Soc., 2017, 139, 7939-7951.

101 Q. Xu, H. Wei, W. Wei, W. Chuirazzi, D. DeSantis, J. Huang and L. Cao, Nucl. Instrum. Methods Phys. Res., Sect. A, 2017, 848, 106-108.

102 A. Xie, C. Hettiarachchi, F. Maddalena, M. E. Witkowski, M. Makowski, W. Drozdowski, A. Arramel, A. T. S. Wee, S. V. Springham, P. Q. Vuong, H. J. Kim, C. Dujardin, P. Coquet, M. D. Birowosuto and C. Dang, Commun. Mater., 2020, 1, 1-10.

103 E. Furuta, Y. Yoshizawa, T. Natake and M. Takiue, Radioisotope, 1997, 46, 912-916.

104 P. Lommatzsch, Am. J. Ophthalmol., 1976, 81, 198-206.

105 T. Maekawa, A. Sumita and S. Makino, J. Nucl. Sci. Technol., 1998, 35, 886-894.

106 J. W. Bae and H. R. Kim, Nucl. Eng. Technol., 2020, 52, 1259-1265. 
107 F. Zhang, C. Herman, Z. He, G. D. Geronimo, E. Vernon and J. Fried, IEEE Trans. Nucl. Sci., 2012, 59, 236-242.

108 G. Yang, H. Mei, Y. T. Guan, G. J. Wang, D. M. Mei and K. Irmscher, J. Phys.: Conf. Ser., 2015, 606, 012013.

109 S. Yakunin, D. N. Dirin, Y. Shynkarenko, V. Morad, I. Cherniukh, O. Nazarenko, D. Kreil, T. Nauser and M. V. Kovalenko, Nat. Photonics, 2016, 10, 585-589.

110 Y. He, W. Ke, G. C. B. Alexander, K. M. McCall, D. G. Chica, Z. Liu, I. Hadar, C. C. Stoumpos, B. W. Wessels and M. G. Kanatzidis, ACS Photonics, 2018, 5, 4132-4138.

111 Y. He, M. Petryk, Z. Liu, D. G. Chica, I. Hadar, C. Leak, W. Ke, I. Spanopoulos, W. Lin, D. Y. Chung, B. W. Wessels, Z. He and M. G. Kanatzidis, Nat. Photonics, 2021, 15, 36-42.

112 Q. Xu, W. Shao, J. Liu, Z. Zhu, X. Ouyang, J. Cai, B. Liu, B. Liang, Z. Wu and X. Ouyang, ACS Appl. Mater. Interfaces, 2019, 11, 47485-47490.

113 N. Kawano, M. Koshimizu, G. Okada, Y. Fujimoto, N. Kawaguchi, T. Yanagida and K. Asai, Sci. Rep., 2017, 7, 14754 .

114 C. W. E. van Eijk, Phys. Med. Biol., 2002, 47, R85-R106.

115 P. K. Spiegel, Am. J. Roentgenol., 1995, 164, 241-243.

116 J. Miao, P. Charalambous, J. Kirz and D. Sayre, Nature, 1999, 400, 342-344.

117 S. Shrestha, R. Fischer, G. J. Matt, P. Feldner, T. Michel, A. Osvet, I. Levchuk, B. Merle, S. Golkar, H. Chen, S. F. Tedde, O. Schmidt, R. Hock, M. Rührig, M. Göken, W. Heiss, G. Anton and C. J. Brabec, Nat. Photonics, 2017, 11, 436-440.

118 Z. Gou, S. Huanglong, W. Ke, H. Sun, H. Tian, X. Gao, $\mathrm{X}$. Zhu, D. Yang and P. Wangyang, Phys. Status Solidi RRL, 2019, 13, 1900094.

119 J. Liu, B. Shabbir, C. Wang, T. Wan, Q. Ou, P. Yu, A. Tadich, X. Jiao, D. Chu, D. Qi, D. Li, R. Kan, Y. Huang, Y. Dong, J. Jasieniak, Y. Zhang and Q. Bao, Adv. Mater., 2019, 31, 1901644.

120 W. Wei, Y. Zhang, Q. Xu, H. Wei, Y. Fang, Q. Wang, Y. Deng, T. Li, A. Gruverman, L. Cao and J. Huang, Nat. Photonics, 2017, 11, 315-321.

121 W. Chen, Y. Liu, Z. Yuan, Z. Xu, Z. Zhang, K. Liu, Z. Jin and X. Tang, J. Radioanal. Nucl. Chem., 2017, 314, 2327-2337.

122 M. D. Birowosuto, D. Cortecchia, W. Drozdowski, K. Brylew, W. Lachmanski, A. Bruno and C. Soci, Sci. Rep., 2016, 6, 37254.

123 J. H. Heo, D. H. Shin, J. K. Park, D. H. Kim, S. J. Lee and S. H. Im, Adv. Mater., 2018, 30, 1801743.

124 Q. Chen, J. Wu, X. Ou, B. Huang, J. Almutlaq, A. A. Zhumekenov, X. Guan, S. Han, L. Liang, Z. Yi, J. Li, X. Xie, Y. Wang, Y. Li, D. Fan, D. B. L. Teh, A. H. All,
O. F. Mohammed, O. M. Bakr, T. Wu, M. Bettinelli, H. Yang, W. Huang and X. Liu, Nature, 2018, 561, 88-93.

125 Y. Zhang, R. Sun, X. Ou, K. Fu, Q. Chen, Y. Ding, L.-J. Xu, L. Liu, Y. Han, A. V. Malko, X. Liu, H. Yang, O. M. Bakr, H. Liu and O. F. Mohammed, ACS Nano, 2019, 13, 2520-2525.

126 H. Zhang, Z. Gao, R. Liang, X. Zheng, X. Geng, Y. Zhao, D. Xie, J. Hong, H. Tian, Y. Yang, X. Wang and T. Ren, IEEE Trans. Electron Devices, 2019, 66, 2224-2229.

127 H. Li, X. Shan, J. N. Neu, T. Geske, M. Davis, P. Mao, K. Xiao, T. Siegrist and Z. Yu, J. Mater. Chem. C, 2018, 6, 11961-11967.

128 Z. Shi, J. Guo, Y. Chen, Q. Li, Y. Pan, H. Zhang, Y. Xia and W. Huang, Adv. Mater., 2017, 29, 1605005.

129 C. Wu, B. Du, W. Luo, Y. Liu, T. Li, D. Wang, X. Guo, H. Ting, Z. Fang, S. Wang, Z. Chen, Y. Chen and L. Xiao, Adv. Opt. Mater., 2018, 6, 1800811.

130 K. M. McCall, Z. Liu, G. Trimarchi, C. C. Stoumpos, W. Lin, Y. He, I. Hadar, M. G. Kanatzidis and B. W. Wessels, ACS Photonics, 2018, 5, 3748-3762.

131 R. Zhuang, X. Wang, W. Ma, Y. Wu, X. Chen, L. Tang, H. Zhu, J. Liu, L. Wu, W. Zhou, X. Liu and Y. (Michael) Yang, Nat. Photonics, 2019, 13, 602-608.

132 X. Zheng, W. Zhao, P. Wang, H. Tan, M. I. Saidaminov, S. Tie, L. Chen, Y. Peng, J. Long and W.-H. Zhang, J. Energy Chem., 2020, 49, 299-306.

133 W. Pan, H. Wu, J. Luo, Z. Deng, C. Ge, C. Chen, X. Jiang, W.-J. Yin, G. Niu, L. Zhu, L. Yin, Y. Zhou, Q. Xie, X. Ke, M. Sui and J. Tang, Nat. Photonics, 2017, 11, 726-732.

134 J. A. Steele, W. Pan, C. Martin, M. Keshavarz, E. Debroye, H. Yuan, S. Banerjee, E. Fron, D. Jonckheere, C. W. Kim, W. Baekelant, G. Niu, J. Tang, J. Vanacken, M. V. der Auweraer, J. Hofkens and M. B. J. Roeffaers, Adv. Mater., 2018, 30, 1804450.

135 F. D. Rossi, J. A. Baker, D. Beynon, K. E. A. Hooper, S. M. P. Meroni, D. Williams, Z. Wei, A. Yasin, C. Charbonneau, E. H. Jewell and T. M. Watson, Adv. Mater. Technol., 2018, 3, 1800156.

136 B. Zat'ko, F. Dubecký, A. Šagátová, K. Sedlačová and L. Ryć, J. Instrum., 2015, 10, C04009.

137 C. Eames, J. M. Frost, P. R. F. Barnes, B. C. O'Regan, A. Walsh and M. S. Islam, Nat. Commun., 2015, 6, 7497.

138 D. Li, H. Wu, H.-C. Cheng, G. Wang, Y. Huang and X. Duan, ACS Nano, 2016, 10, 6933-6941.

139 L. Bertoluzzi, J. B. Patel, K. A. Bush, C. C. Boyd, R. A. Kerner, B. C. O'Regan and M. D. McGehee, Adv. Energy Mater., 2021, 11, 2002614.

140 C. C. Boyd, R. Cheacharoen, T. Leijtens and M. D. McGehee, Chem. Rev., 2019, 119, 3418-3451. 